TRANSACTIONS OF THE

AMERICAN MATHEMATICAL SOCIETY

Volume 361, Number 4, April 2009, Pages 2181-2206

S 0002-9947(08)04798-3

Article electronically published on November 25, 2008

\title{
RELATIVELY INHERENTLY NONFINITELY Q-BASED SEMIGROUPS
}

\author{
MARCEL JACKSON AND MIKHAIL VOLKOV
}

\begin{abstract}
We prove that every semigroup $\mathbf{S}$ whose quasivariety contains a 3 -nilpotent semigroup or a semigroup of index more than 2 has no finite basis for its quasi-identities provided that one of the following properties holds:

- $\mathbf{S}$ is finite;

- $\mathbf{S}$ has a faithful representation by injective partial maps on a set;

- $\mathbf{S}$ has a faithful representation by order preserving maps on a chain. As a corollary it is shown that, in an asymptotic sense, almost all finite semigroups and finite monoids admit no finite basis for their quasi-identities.
\end{abstract}

\section{BACKGROUND AND MOTIVATION}

Recall that a semigroup quasi-identity is a sentence

$$
(\forall \bar{x})\left[\left(p_{1}(\bar{x}) \approx q_{1}(\bar{x}) \& \cdots \& p_{n}(\bar{x}) \approx q_{n}(\bar{x})\right) \rightarrow p(\bar{x}) \approx q(\bar{x})\right]
$$

where $n$ is nonnegative integer and each $p_{i}(\bar{x})$ and $q_{i}(\bar{x})$ are semigroup words in the variables $\bar{x}=x_{1}, \ldots, x_{m}$. Note that a semigroup identity

$$
(\forall \bar{x})(p(\bar{x}) \approx q(\bar{x}))
$$

is a semigroup quasi-identity; this corresponds to the situation where $n=0$. We adopt the usual convention of omitting the universal quantifiers from quasiidentities.

Standard examples of semigroup quasi-identities are the cancellation laws

$$
x y \approx x z \rightarrow y \approx z \text { and } y x \approx z x \rightarrow y \approx z ;
$$

however there are many other familiar properties described by quasi-identities. For example, the statement that idempotents related by Green's $\mathcal{J}$-relation cannot form a nontrivial subsemilattice can be written as

$$
x e y \approx f \& u f v \approx e \& e^{2} \approx e \& f^{2} \approx f \& e f \approx e \& f e \approx e \rightarrow e \approx f .
$$

This quasi-identity is satisfied by all finite semigroups, but fails, say, on the bicyclic semigroup.

Received by the editors June 4, 2007.

2000 Mathematics Subject Classification. Primary 08C15, 20M20.

Key words and phrases. Quasi-identity, quasivariety, universal class, semigroup, injective map, order preserving map, finite q-basis property, inherently nonfinitely q-based semigroup relative to a class, 3-nilpotent semigroup, homotopy embedding.

The first author was supported by ARC Discovery Project Grant DP0342459.

The second author acknowledges support from the Russian Foundation for Basic Research, grants 05-01-00540 and 06-01-00613. The paper was initiated during the second author's Distinguished Fellowship at the Institute for Advanced Study of La Trobe University.

(C)2008 American Mathematical Society Reverts to public domain 28 years from publication 
The quasivariety $\mathbb{Q}(\mathcal{K})$ generated by a class $\mathcal{K}$ of semigroups is the class of all models of the quasi-identities satisfied by $\mathcal{K}$. Equivalently, $\mathbb{Q}(\mathcal{K})$ is the set of all isomorphic copies of subsemigroups of (possibly empty) direct products of ultraproducts of members of $\mathcal{K}$; in symbols $\mathbb{Q}(\mathcal{K})=\mathbb{I S P P} \mathbb{u}_{\mathrm{u}}(\mathcal{K})$. This is a semigroup case of a classic result by Mal'cev [16] dealing with arbitrary algebras; a proof of Mal'cev's result can be found in any standard text on universal algebra; see 1 , $\S \mathrm{V} .2]$ for example.

The $\mathbb{I S P P}$ u characterisation of quasivarieties readily implies that whenever $Q$ is a quasivariety of algebras of some similarity type, and $\mathcal{P}$ is the class of subreducts of $\mathcal{Q}$ (that is, subalgebras of reducts of members of $\mathcal{Q}$ to some smaller type), then $\mathcal{P}$ is a quasivariety. For example, the class of all inverse semigroups (in the operations $\cdot,^{-1}$ ) is a quasivariety (a variety even), so the class of semigroup subreducts of inverse semigroups is a quasivariety, which we will denote by $\mathcal{J}$.

Every inverse semigroup is faithfully representable as a system of injective partial maps on some set, under the operations of composition and inverse. So every member of $\mathcal{J}$ is representable as a system of injective partial maps on some set under the operation of composition. Conversely, every semigroup representable as injective partial maps on a set $X$ embeds into the semigroup of all injective partial maps on $X$, which is the semigroup reduct of the full symmetric inverse semigroup. So $\mathcal{J}$ is precisely the class of semigroups that are representable as injective partial maps on some set. In the same way, the reader will see that the class of all semigroups representable as a system of permutations on a set (let us call this $\mathcal{G}$ ), is the same as the semigroup subreducts of the variety of groups, or equivalently the class of semigroups embeddable in a group. As these examples demonstrate, quasivarieties arise frequently in the study of representations of algebraic structures by way of particular kinds of maps (in the cases above, as injective partial maps and by permutations on a set respectively); see [29] and [10] for surveys of this rich area.

When considering a naturally defined quasivariety $\mathcal{Q}$ such as the quasivarieties $\mathcal{J}$ and $\mathcal{G}$, the fundamental question is to find its characterisation by some explicit quasi-identity basis $\Sigma$. Such a characterisation says that a semigroup $\mathbf{S}$ lies in $\mathcal{Q}$ if and only if it satisfies every quasi-identity in $\Sigma$. A quasivariety $\mathcal{Q}$ that admits a finite quasi-identity basis is said to be finitely q-based; in the opposite case, $Q$ is said to be nonfinitely q-based. Of course, for quasivarieties the property of being finitely/nonfinitely q-based coincides with the property of being finitely/nonfinitely axiomatised in first order logic. We shall say that a class $\mathcal{K}$ is finitely/nonfinitely q-based if the quasivariety $\mathbb{Q}(\mathcal{K})$ is. (A small warning: the previous remark does not apply to this general situation since the class $\mathcal{K}$ may have no first order axiomatisation at all, but even if $\mathcal{K}$ is finitely axiomatised in the first order logic - if $\mathcal{K}$ consists of a single finite semigroup, say - it may well be nonfinitely q-based.)

Returning to our basic examples, the quasivarieties $\mathcal{J}$ and $\mathcal{G}$, we recall that the quasivariety $\mathcal{G}$ was famously characterised by $\mathrm{Mal}^{\prime} \mathrm{cev}$ [14, 15], who also showed that it admits no finite axiomatisation (see [3, Ch. 12] for a detailed account of Mal'cev's results). The quasivariety J was characterised by Schein [26, 27, 28. The complicated history of the publication of these results and their proofs is detailed in Schein [30, where one can find, with complete proofs, his elegant structural characterisation (see Lemma 2.1 below), an infinite set of quasi-identities defining $\mathcal{J}$, and a proof that no finite set of quasi-identities is sufficient to characterise $\mathcal{J}$. The first of these results can also be found in [3, Ch. 7], while the second result 
follows in a standard way from the first. The third step is the most technical, and one of the contributions of this article is to give a new, very easy proof.

Another example of an abstract class of semigroups representable in some particular kind of maps, is the class $\mathcal{O}$ of semigroups representable as order preserving transformations of a chain. The class $\mathcal{O}$ is also easily seen to be a quasivariety, and was first proved to have no finite axiomatisation by Vernitskiì 34. A (necessarily infinite) axiomatisation of it was given by Repnitskiǐ and Vernitskiǐ 22, who also gave an elegant structural characterisation of membership in $\mathcal{O}$. Again, in this article we provide a new, very easy proof that $\mathcal{O}$ is nonfinitely q-based.

The quasivarieties generated by individual finite semigroups have also been studied. Ol'shanskiǐ [20] showed that a finite group generates a quasivariety with a finite quasi-identity basis if and only if all of its Sylow subgroups are abelian. (This sharply contrasts with a well-known result by Oates and Powell [19] who proved that every finite group has a finite basis for its identities.) In fact Ol'shanskiĩ showed that a group $\mathbf{G}$ with a nonabelian Sylow subgroup is strongly nonfinitely $q$-based (SNFQB): any finite set of finite algebras whose quasivariety contains $\mathbf{G}$ has no finite basis for its quasi-identities. Sapir [25] proved the same for completely simple semigroups whose idempotents do not form a subsemigroup 11 Combined with Ol'shanski1's result, this shows that a finite simple semigroup $\mathbf{S}$ is finitely qbased if and only if every subgroup of $\mathbf{S}$ has only abelian Sylow subgroups and the idempotents of $\mathbf{S}$ form a subsemigroup. Furthermore, a nonfinitely q-based finite simple semigroup is SNFQB.

An even stronger version of the SNFQB property is the property of being inherently nonfinitely q-based (INFQB): a locally finite quasivariety $\mathcal{Q}$ (or an algebra generating such a quasivariety) is called INFQB if every locally finite quasivariety containing $Q$ is not finitely q-based. For semigroups, the INFQB property turns out to be too restrictive, as Margolis and Sapir [17] showed that there are no INFQB finite semigroups. On the other hand, Lawrence and Willard 13] constructed some finite INFQB unary semigroups (that is, semigroups equipped with an additional unary operation); these remain the only known examples of finite INFQB algebras, although partial algebras with this property are more easily constructed; see 8 .

We suggest the following general framework in which the notions of being inherently or strongly nonfinitely q-based fit rather naturally. Let $\mathscr{P}$ be a property of quasivarieties of semigroups, such as "local finiteness", or "being generated by a finite set of finite semigroups". We will say that a class $\mathcal{K}$ of semigroups is inherently nonfinitely q-based relative to $\mathscr{P}$ (abbreviated INFQB relative to $\mathscr{P}$ ) if

- $\mathcal{K}$ generates a quasivariety satisfying $\mathscr{P}$ and

- whenever a class of semigroups $\mathcal{J}$ generates a quasivariety satisfying $\mathscr{P}$ and containing $\mathcal{K}$, then $\mathcal{J}$ has no finite basis of quasi-identities.

The usual notion of INFQB coincides with INFQB with respect to local finiteness, while SNFQB corresponds to INFQB with respect to being generated by a finite set of finite algebras. In the present paper we will exhibit a plethora of examples

\footnotetext{
${ }^{1}$ This result is essentially proved by Sapir in the more readily available article [23]; however, there only the nonfinite q-basis property is claimed rather than the full strong nonfinitely q-basis property. The proof in 23. yields the latter property after almost trivial modifications: simply replace the number $|S|$ at the bottom of page 76 in 23 by any larger number $p$. A more detailed presentation of the second half of this proof can be found in the first author's article [9].
} 
of semigroups which are INFQB with respect to the following natural properties of quasivarieties:

$\mathscr{I}$ : to be contained in the quasivariety $\mathcal{J}$;

$\mathscr{O}$ : to be contained in the quasivariety $\mathcal{O}$;

$\mathscr{F}$ : to be contained in a quasivariety generated by a finite set of finite semigroups.

This constitutes a vast generalisation of the aforementioned results concerning the quasi-identity bases of $\mathcal{J}, \mathcal{O}$, etc. In fact, our methods are powerful enough to show that, in an asymptotic sense, almost all finite semigroups and finite monoids are strongly nonfinitely q-based.

In the next section we present our results in more detail and give a general overview of the article.

\section{MAin RESUlts}

1.1. Preliminaries. We first recall some basic notions. For more details on semigroups consult an introductory text such as [3. For an easy introduction to some model-theoretic concepts, see [1, Chapter V], and for a detailed analysis of the theory of quasivarieties consult [6].

A semigroup is said to be $k$-nilpotent if it satisfies the identity $x_{1} \cdots x_{k} \approx$ $y_{1} \cdots y_{k}$. Equivalently, a $k$-nilpotent semigroup is a semigroup containing a (multiplicative) 0 element and such that any product of length $k$ or more equals 0 (so $x_{1} \cdots x_{k} \approx y_{1} \cdots y_{k}$ is often written as $\left.x_{1} \cdots x_{k} \approx 0\right)$. A semigroup is said to be nilpotent if it is $k$-nilpotent for some $k \in \mathbb{N}$. We let $\mathcal{N}_{k}$ denote the class of all $k$-nilpotent semigroups, and say that a semigroup is a proper $k$-nilpotent semigroup if it lies in $\mathcal{N}_{k} \backslash \mathcal{N}_{k-1}$. A monoid obtained by adjoining an identity element to a $k$-nilpotent semigroup is said to be a $k$-nilpotent monoid.

For each $i, p \in \mathbb{N}$, we let $\mathbf{C}_{i, p}$ denote the monogenic semigroup with the presentation $\left\langle a \mid a^{i}=a^{i+p}\right\rangle$. We let $\theta_{i, p}$ denote the congruence on the semigroup $\langle\mathbb{N} ;+\rangle$ defined by $j \equiv k \bmod \theta_{i, p}$ if $j=k$ or both $j, k \geq i$ and $j \equiv k \bmod p$. So $\mathbf{C}_{i, p} \cong\langle\mathbb{N} ;+\rangle / \theta_{i, p}$ under the map $a^{j} \mapsto j$. The two-element null semigroup is $\mathbf{C}_{2,1}$, while $\mathbf{C}_{3,1}$ is a proper 3-nilpotent semigroup. We call the number $i$ the index of the monogenic semigroup $\mathbf{C}_{i, p}$; by the index of a finite semigroup we mean the maximum index of its monogenic subsemigroups.

Recall that a universal sentence (for semigroups) is a first order sentence of the form

$$
\forall x_{1} \forall x_{2} \ldots \forall x_{n} \Phi\left(x_{1}, \ldots, x_{n}\right)
$$

where $\Phi=\Phi\left(x_{1}, \ldots, x_{n}\right)$ is a quantifier-free formula in the first order language of semigroups whose variables are precisely $x_{1}, \ldots, x_{n}$ (for some $n$ ). Note that $\Phi$ is simply a well-formed expression built from the Boolean connectives $\&, \vee, \neg, \rightarrow$ and atomic formulæ $u \approx v$ (where $u$ and $v$ are semigroup words). Universal sentences include as special cases, quasi-identities (when $\Phi$ is an implication of the form described at the start of the article) and identities (when $\Phi$ is an atomic formula). A universal class is a class of semigroups equal to the class of all models of some set of universal sentences $\Sigma$. It is well known that universal classes can be equivalently characterised as those classes closed under the formation of ultraproducts and taking subsemigroups. Universal classes include as special cases quasivarieties (when they are closed under arbitrary direct products) and varieties (when they are quasivarieties and closed under homomorphic images). 
1.2. Easy proofs. The results in the article fall into two parts. Our first contribution is to give new, easy proofs that the classes $\mathcal{J}$ (Section 2) and $\mathcal{O}$ (Section 3) are not finitely axiomatised. These results are by far superceded by our main theorem, however can be read alone and, as we believe, give the shortest way to demonstrate the nonfinite axiomatisability of some important classes of semigroups.

In the case of J, Schein's proof in 28, 30 is similar to ours except based around a known basis for $\mathcal{J}$ (also found by Schein). Because the basis is quite complicated, the corresponding nonfinite axiomatisability proof is also complicated. Our proof concentrates on a particularly simple system of quasi-identities and uses 3-nilpotent semigroups, which are very easy to work with since associativity comes "for free". An example of a 3-nilpotent semigroup not in $\mathcal{J}$ is given by Schein in [31; our result shows that the intersection of $\mathcal{J}$ with the class of 3 -nilpotent semigroups is "infinitely jagged".

We then turn to the class $\mathcal{O}$ and use the same method to obtain a new proof of the nonfinite axiomatisability result of Vernitskiir. Vernitskiî's original proof is similar in style to ours, but our use of 3-nilpotent semigroups makes the proof particularly transparent.

1.3. The main theorem. The main result of the article is the following theorem showing, in particular, that proper 3-nilpotent semigroups and finite semigroups of index more than 2 are INFQB relative to each of the properties $\mathscr{I}, \mathscr{O}$ or $\mathscr{F}$ mentioned at the end of the introductory section.

Theorem 1.1. Let $\mathcal{U}$ be any universal class that contains either the quasivariety of some proper 3-nilpotent semigroup or the quasivariety of some finite semigroup of index more than 2. If one of the following conditions holds:

(i): $\mathcal{U}$ is contained in the quasivariety $\mathcal{J}$ of all semigroups having a faithful representation by injective partial maps on a set,

(ii): $\mathcal{U}$ is contained in the quasivariety $\mathcal{O}$ of all semigroups having a faithful representation by order preserving partial maps on a chain,

(iii): $\mathcal{U}$ is contained in the quasivariety $\mathcal{F}_{k}$ generated by all $k$-element groupoids 2 for any fixed positive integer $k$,

then $\mathcal{U}$ has no finite axiomatisation in first order logic.

The same statement holds in the type $\langle 2,0\rangle$ for 3-nilpotent monoids and finite monoids of index greater than 2 (after an obvious adjustment to the definitions of $\mathcal{J}, \mathcal{O}$ and $\left.\mathcal{F}_{k}\right)$.

The result is rather surprising since it shows that virtually any universal class $\mathcal{U}$ of semigroups representable in a "nice" way (as injective partial maps or as order preserving maps) will admit no "nice" first order characterisation, and also that two natural finiteness conditions - finite axiomatisability and finite generationare virtually incompatible. Theorem 1.1 radically extends some earlier works and has many interesting corollaries. We discuss some of these corollaries in the next two subsections while here we want to comment on the conditions appearing in the formulation of the theorem.

First of all, we observe that the two conditions "to contain the quasivariety of some proper 3-nilpotent semigroup" and "to the quasivariety of some finite semigroup of index more than 2" are independent in general. Indeed, on the one hand,

\footnotetext{
${ }^{2}$ Here a groupoid is any algebra with a single binary operation.
} 
if we define a multiplication on the set $\{a, b, c, 0\}$ by putting $a b=c$ and letting all other products be equal to 0 , we get a proper 3-nilpotent semigroup satisfying the identity $x^{2} \approx x^{3}$. Therefore in the quasivariety (and even in the variety) of this semigroup, the index of each finite semigroup is at most 2 . On the other hand, it is easy to check that the monogenic semigroup $\mathbf{C}_{4,1}$ satisfies the quasi-identity

$$
x^{3} \approx x^{4} \& y^{3} \approx y^{4} \rightarrow x y \approx(x y)^{2},
$$

which fails in every proper 3-nilpotent semigroup.

To prove Theorem 1.1 it basically suffices to prove the relative INFQB property for a quasivariety that is minimal with respect to containing either some proper 3nilpotent semigroup or some monogenic semigroup of index more than 2 and lying in either $\mathcal{J}$ or $\mathcal{O}$ or $\mathcal{F}_{k}$ for some $k$. Unfortunately, it is possible to show that, with respect to the properties of interest, there are up to three minimal quasivarieties of 3-nilpotent semigroups, and two infinite families of minimal quasivarieties of monogenic semigroups (the index 3 and index 4 cases). In order to overcome this difficulty, in Section 4 we give a general method for translating the nonfinite qbasis properties of 3-nilpotent semigroups. This method, which is based on the concept of a homotopy embedding, enables us to avoid the separate consideration of proper 3-nilpotent semigroups and appears to be of some independent interest. In Section 5 we analyse some particular semigroups in the quasivariety of a monogenic semigroup and provide a method of translating many nonfinite q-basis properties of the proper 3-nilpotent semigroup $\mathbf{C}_{3,1}$ to arbitrary finite monogenic semigroups $\mathbf{C}_{i, p}$ with $i \geq 3$.

The formulation of Theorem 1.1 suggests that its proof should be split into 3 parts corresponding to the conditions (i), (ii), and (iii), but in fact we have managed to unify the two latter cases. Let $\vee$ denote the quasivariety join; that is, for any two quasivarieties $\mathcal{P}$ and $\mathcal{Q}$, the least quasivariety containing both $\mathcal{P}$ and $\mathcal{Q}$ is denoted $\mathcal{P} \vee \mathcal{Q}$. Our unified proof applies to any universal class $\mathcal{U}$ that contains either the quasivariety of some proper 3-nilpotent semigroup or the quasivariety of some finite semigroup of index more than 2 provided the following condition holds:

(ii) $\vee$ (iii): $\mathcal{U}$ is contained in the quasivariety $\mathcal{O} \vee \mathcal{F}_{k}$.

We were not able, however, to find a unified proof which would, in a similar way, cover all the cases (i)-(iii) at once.

The main details of the proof of Theorem $1.1_{\text {i) }}$ are presented in Section 6 while Section 7 is devoted to the proof of Theorem $1.1_{(i i) \vee(i i i)}$. The monoid version of the main theorem is discussed in Section 8 .

1.4. Some corollaries. Theorem 1.1 $_{\mathrm{i})}$ extends recent work by Cowan, Reilly, Trotter and the second author [4. In the terminology of the present article, they showed that the class $\mathcal{B}$ consisting of semigroup subreducts of the inverse semigroup variety generated by the 6 -element Brandt monoid $\mathbf{B}_{2}^{1}$ is INFQB relative to membership in J. This result follows from Theorem $1.1_{\text {i) }}$ since $\mathcal{B}$ contains the Brandt semigroup $\mathbf{B}_{3}$ and the latter possesses a proper 3 -nilpotent subsemigroup. (Indeed, using the representation of $\mathbf{B}_{3}$ as a matrix semigroup consisting of all $3 \times 3$ matrix units $e_{i j}, 1 \leq i, j \leq 3$, and the $3 \times 3$ zero matrix 0 , one readily sees that the subsemigroup on $\left\{e_{12}, e_{23}, e_{13}, 0\right\}$ is a proper 3 -nilpotent semigroup.) Observe that no finite semigroup generates the quasivariety $\mathcal{B}$ since this class contains subdirectly irreducible algebras of arbitrary cardinality (for example, the Brandt semigroups 
$\mathbf{B}_{\kappa}$ for any cardinal $\left.\kappa\right)$. Thus, in contrast to Theorem 1.1 ${ }_{\mathrm{i})}$, the cited result from [4] does not produce any finite semigroup being INFQB relative to membership in $\mathcal{J}$.

We can also obtain the following corollary of Theorem 1.1 i) $_{\text {) }}$ (here recall that a Clifford semigroup is a semilattice of groups, or equivalently an inverse semigroup whose idempotent elements are central).

Proposition 1.2. Let $\mathcal{V}$ be a quasivariety of inverse semigroups and $\mathcal{V}^{b}$ the class of semigroup subreducts of $\mathcal{V}$. Then $\mathcal{V}^{b}$ generates a nonfinitely based quasivariety of semigroups provided that $\mathcal{V}$ contains the inverse Brandt semigroup $\mathbf{B}_{3}$. If $\mathcal{V}$ is a finitely generated variety of inverse semigroups, then $\mathcal{V}^{b}$ generates a finitely based quasivariety of semigroups if and only if it is a variety of Clifford semigroups.

Proof. The first statement is a restriction of Theorem $1.1_{\text {i) }}$ because inverse semigroups are representable as semigroups of injective partial maps and because the Brandt semigroup $\mathbf{B}_{3}$ contains a proper 3-nilpotent subsemigroup.

Let $\mathcal{V}$ be a finitely generated variety of inverse semigroups, and $\mathcal{V}^{b}$ be the semigroup reducts of members of $\mathcal{V}$. Now $\mathbf{B}_{3}$ is not a Clifford semigroup. So by the first statement of this theorem, it will suffice to show that if $\mathbf{B}_{3} \notin \mathcal{V}$, then $\mathcal{V}$ is a variety of Clifford semigroups and $\mathcal{V}^{b}$ generates a finitely based quasivariety of semigroups. The first condition is very well known; see [21] for example. Now, as $\mathcal{V}$ is a finitely generated variety of inverse Clifford semigroups there is a number $d$ such that the identity $\mathcal{V} \models x^{-1} \approx x^{d}$. Hence a member of $\mathcal{V}$ is term equivalent to its semigroup reduct in $\mathcal{V}^{b}$, and hence $\mathcal{V}^{b}$ is a variety of semigroups generated by a finite set of Clifford semigroups. It is again well known that such a variety is finitely based (as a variety, and hence also as a quasivariety); see 32 for example.

Theorem $1.1 n_{\text {ii) } \vee(i i i)}$ also has many corollaries of which we mention only two here. The first corollary makes an interesting complement to Sapir's theorem on quasiidentities of finite simple semigroups mentioned in the introductory section. Recall that a finite simple semigroup always has index 1, and hence, Sapir's result showed that semigroups of index 1 are frequently SNFQB.

Corollary 1.3. A finite semigroup or monoid is $S N F Q B$ provided that it has index greater than 2 or it contains a proper 3-nilpotent subsemigroup.

The question of deciding when a finite semigroup has a finite basis for its quasiidentities constitutes a well-known open problem. Corollary 1.3 shows that the problem is equivalent to its restriction to semigroups of index 1 or 2 . In particular, we have the following corollary.

Corollary 1.4. A finite nilpotent semigroup has a finite q-basis if and only if it is a null semigroup.

Proof. A null semigroup has a finite basis for its quasi-identities: in fact the single identity $x y \approx u v$ is a basis for the quasi-identities of a nontrivial null semigroup. On the other hand, a finite nilpotent semigroup $\mathbf{S}$ that is not a null semigroup either has index more than 2, or contains a proper 3-nilpotent subsemigroup, and hence is SNFQB by Corollary 1.3 .

We mention that the particular 3-nilpotent semigroup $\mathbf{C}_{3,1}$ was proved to be nonfinitely q-based by Sapir [23] (moreover he showed that it has no independent basis of quasi-identities), while Clark, Davey, Jackson and Pitkethly [2] showed that $\mathbf{C}_{i, p}$ is nonfinitely q-based if and only if $i>2$. 
1.5. Almost all finite semigroups have no finite quasi-identity basis. This is, probably, the most interesting consequence of Theorem $1.1_{\text {ii) } \vee(i i i)}$, which is, as we shall explain, rather unexpected. Let us first define what we mean by "almost all".

For some property $\mathscr{P}$ of algebras, and a variety $\mathcal{V}$ let $\mathscr{P}_{\mathcal{V}}(n)$ denote the fraction of all $\mathcal{V}$-algebras on the set $\{0,1, \ldots, n-1\}$ with property $\mathscr{P}$, if the fraction exists, and let $\mathscr{P}_{\mathcal{V}}(n)=1$ otherwise. Let $\mathscr{F} \mathscr{B}$ denote the finite basis property and let $\mathscr{F} \mathscr{Q} \mathscr{B}$ denote the finite q-basis property. Murskii [18] showed that

$$
\lim _{n \rightarrow \infty} \mathscr{F} \mathscr{B} \mathcal{V}_{\mathcal{F}}(n)=1 \text {, }
$$

when $\mathcal{V}_{\mathcal{F}}$ is the variety of all algebras of some finite signature $\mathcal{F}$. In this sense, almost all finite algebras are finitely based. The corresponding results

$$
\lim _{n \rightarrow \infty} \mathscr{F}_{\text {Sgp }}(n)=\lim _{n \rightarrow \infty} \mathscr{F} \mathscr{B}_{\text {Mon }}(n)=1
$$

for the variety Sgp of all semigroups and the variety Mon of all monoids (in the type $\langle 2,0\rangle)$ are observed in [7, 35]; so semigroups and monoids behave quite "typically" with respect to the axiomatisability properties of their identities.

In fact, Murskiř's results in [18] also imply that

$$
\lim _{n \rightarrow \infty} \mathscr{F} \mathscr{Q} \mathscr{B} v_{\mathcal{F}}(n)=1,
$$

provided $\mathcal{F}$ contains an operation of arity more than 1 . Murskiĩ shows that almost all finite $\mathcal{F}$-algebras $\mathbf{A}$ have a discriminator function

$$
t(x, y, z):= \begin{cases}z & \text { if } x=y \\ x & \text { if } x \neq y\end{cases}
$$

as a term function. Now we recall some basic universal algebra; see [1] for the details. The existence of a discriminator term function on $\mathbf{A}$ implies that the variety $\mathbb{V}(\mathbf{A})$ generated by $\mathbf{A}$ is congruence distributive, and hence $\mathbf{A}$ has a finite identity basis by Baker's Finite Basis Theorem. By Jónsson's Lemma, the subdirectly irreducibles in the variety $\mathbb{V}(\mathbf{A})$ all lie in $\mathbb{H S P}_{\mathrm{u}}(\mathbf{A})=\mathbb{H} \mathbb{S}(\mathbf{A})$. However every subalgebra of an algebra with a discriminator term is congruence-free, so that $\mathbb{H} \mathbb{S}(\mathbf{A})=\mathbb{S}(\mathbf{A}) \cup \mathbb{I}(\mathbf{1})$, where $\mathbf{1}$ is the one element algebra of type $\mathcal{F}$. Now recall that every algebra in $\mathbb{V}(\mathbf{A})$ is a subdirect product of subdirectly irreducible members of $\mathbb{V}(\mathbf{A})$. We find that $\mathbb{V}(\mathbf{A})=\mathbb{I S P}(\mathbb{S}(\mathbf{A}) \cup \mathbb{I}(\mathbf{1}))=\mathbb{I S P}(\mathbf{A})=\mathbb{Q}(\mathbf{A})$ (note that $\mathbf{1}$ arises as the empty power of $\mathbf{A})$. So the quasivariety of $\mathbf{A}$ is also finitely axiomatisable.

In contrast, our next result shows that semigroups and monoids are in fact atypical with respect to satisfaction of the finite q-basis property.

Proposition 1.5. $\lim _{n \rightarrow \infty} \mathscr{F} \mathscr{Q} \mathscr{B}_{\mathrm{Sgp}}(n)=\lim _{n \rightarrow \infty} \mathscr{F} \mathscr{Q} \mathscr{B}_{\mathrm{Mon}}(n)=0$.

Proof. Let $\mathscr{T}$ be the property of being a proper 3-nilpotent semigroup, and $\mathscr{T}^{1}$ be the property of being a proper 3-nilpotent semigroup with an adjoined identity element. Kleitman, Rothschild and Spencer [11] showed that $\lim _{n \rightarrow \infty} \mathscr{T}_{\text {Sgp }}(n)=1$, while Koubek and Rödl $\left[12\right.$ proved that $\lim _{n \rightarrow \infty} \mathscr{T}_{\text {Mon }}^{1}(n)=1$. The claim now follows because a proper 3-nilpotent semigroup, or the monoid obtained from such a semigroup by adjoining an identity element, is SNFQB (Corollary 1.3). Thus, almost all finite semigroups [monoids] are nonfinitely q-based. 
1.6. Two general lemmas. We conclude this section by recalling two simple general facts, which will be very often used in the sequel.

In the introductory section, we mentioned Mal'cev's formula $\mathbb{Q}(\mathcal{K})=\mathbb{I S P P} \mathbb{u}_{\mathrm{u}}(\mathcal{K})$ for the quasivariety generated by a given class $\mathcal{K}$. In the case where $\mathcal{K}$ is a finite class of finite semigroups, or more generally, when $\mathcal{K}$ is closed under taking ultraproducts (up to isomorphism), we have $\mathbb{I S P P}_{\mathrm{u}}(\mathcal{K})=\mathbb{I S P}(\mathcal{K})$ and then we can use projection maps to obtain the following handy characterisation of $\mathbb{Q}(\mathcal{K})$.

Lemma 1.6. Let $\mathcal{K}$ be a class of similar algebras closed under taking ultraproducts: $\mathbb{P}_{\mathrm{u}}(\mathcal{K}) \subseteq \mathbb{I}(\mathcal{K})$. An algebra $\mathbf{A}$ is contained in the quasivariety generated by $\mathcal{K}$ if and only if for every $a, b \in A$ with $a \neq b$ there is $\mathbf{B} \in \mathcal{K}$ and a homomorphism $\phi: \mathbf{A} \rightarrow \mathbf{B}$ with $\phi(a) \neq \phi(b)$.

The following approach is used in all of our proofs of nonfinite axiomatisation (it goes back to Mal'cev, see [15]). Suppose we have a particular quasivariety $\mathcal{Q}$ of interest (J for example). We also fix some "small" quasivariety $\mathcal{J} \subseteq \mathcal{Q}$ (for example $\mathcal{J} \cap \mathcal{N}_{3}$ ). Then we construct, for each $n \in \mathbb{N}$, a semigroup $\mathbf{S}_{n}$ with the property that $\mathbf{S}_{n}$ is not contained in $\mathcal{Q}$, while $n$-generated subsemigroups of $\mathbf{S}_{n}$ are contained in $\mathcal{\partial}$. As we now state in lemma form, this is all that is needed.

Lemma 1.7. Let $\mathcal{J}, \mathcal{Q}$ be quasivarieties and $\mathcal{K}$ be a class with $\mathcal{J} \subseteq \mathcal{K} \subseteq \mathcal{Q}$. If, for each $n \in \mathbb{N}$ there are semigroups $\mathbf{S}_{n}$ with $\mathbf{S}_{n} \notin \mathcal{Q}$ but such that n-generated subsemigroups of $\mathbf{S}_{n}$ are contained in $\mathcal{J}$, then $\mathcal{K}$ has no finite axiomatisation in first order logic. In particular, if $\mathcal{K}$ is a quasivariety, then it has no finite basis for its quasi-identities.

Proof. We may assume that $\mathcal{K}$ is an elementary class, since otherwise it has no axiomatisation in first order logic. Take an ultraproduct $\mathbf{S}:=\prod_{\mathcal{U}}\left\{\mathbf{S}_{n} \mid n \in \mathbb{N}\right\}$ over a nonprincipal ultrafilter $\mathcal{U}$. Then $\mathbf{S}$ satisfies the universal theory of $\mathcal{J}$; that is, $\mathbf{S} \in \mathcal{J} \subseteq \mathcal{K}$. Since $\mathbf{S}_{n} \notin \mathcal{K}$ for any $n$, this shows that $\mathcal{K}$ is not finitely axiomatisable (see [1, §V.2] for example).

\section{Part 1. Short proofs.}

\section{J IS NOT FINITELY AXIOMATISABLE}

The result stated in the section title was first proved by Schein [28]; see also [30]. In this section we give a new easy proof of it. The proof makes use of Schein's first characterisation of membership in $\mathcal{J}$.

Let a subset $H$ of a semigroup $\mathbf{S}$ be called strong if for $u, v, x, y \in S^{1}$ we have $x v, u v, u y \in H$ implies $x y \in H$, whenever these products make sense (note that we cannot have, say, $u=v=1$ unless $\mathbf{S}^{1}=\mathbf{S}$ because $u v \in H \subseteq S$ ). On a semigroup $\mathbf{S}$, let $\mathcal{T}_{\mathbf{S}}$ denote the family of all strong subsets. Appealing to a topological analogy, we say that $\mathbf{S}$ is $T_{0}$ with respect to $\mathcal{T}_{\mathbf{S}}$ if for every pair of distinct elements $a, b \in S$ there exists $H \in \mathcal{T}_{\mathbf{S}}$ with $|H \cap\{a, b\}|=1$. The main tool is the following result due to Schein.

Lemma 2.1 (Schein [27]). A semigroup $\mathbf{S}$ lies in $\mathcal{J}$ if and only if it is $T_{0}$ with respect to $\mathcal{T}_{\mathbf{S}}$.

We are going to use Lemma 2.1 to prove that $\mathcal{J}$ has no finite basis for its quasiidentities. The proof follows the template described by Lemma 1.7, with $\mathcal{J}:=\mathcal{N}_{3} \cap \mathcal{J}$ and $Q:=\mathcal{J}$. 
For each $n \in \mathbb{N}$ define a semigroup $\mathbf{S}_{n}$ on the set

$$
\{0\} \cup\left\{w_{i} \mid i \in\{0,1, \ldots, 2 n\}, w \in\{a, b, c, d, e\}\right\}
$$

with the multiplication

$$
\begin{aligned}
& a_{i} c_{i}=b_{i} c_{i}=b_{i} d_{i}=e_{i}, \\
& a_{i} d_{i}=e_{i+1}
\end{aligned}
$$

\begin{tabular}{|c|c|c|c|c|c|c|}
\hline & $c_{0}$ & $d_{0}$ & $c_{1}$ & $d_{1}$ & $c_{2 n}$ & $d_{2 n}$ \\
\hline$a_{0}$ & $e_{0}$ & $e_{1}$ & & & & \\
\hline$b_{0}$ & $e_{0}$ & $e_{0}$ & & & & \\
\hline$a_{1}$ & & & $e_{1}$ & $e_{2}$ & & \\
\hline$b_{1}$ & & & $e_{1}$ & $e_{1}$ & & \\
\hline$a_{2 n}$ & & & & & $e_{2 n}$ & $e_{0}$ \\
\hline$b_{2 n}$ & & & & & $e_{2 n}$ & $e_{2 n}$ \\
\hline
\end{tabular}

(where addition in the subscript is taken modulo $2 n+1$ ) and all other products are 0 . The following table gives the nonzero values of the product.

It is clear that any product $(x y) z$ or $x(y z)$ equals 0 in $\mathbf{S}_{n}$, so $\mathbf{S}_{n}$ is a semigroup in $\mathcal{N}_{3}$. However $\mathbf{S}_{n}$ is not in $\mathcal{J}$, since any strong subset containing $e_{i}$ also contains $e_{i+1}$, and hence all of $\left\{e_{0}, \ldots, e_{2 n}\right\}$. So we cannot separate $e_{0}$ and $e_{1}$ by a strong subset, and Lemma 2.1 applies.

Let $\alpha$ denote the automorphism of $\mathbf{S}_{n}$ defined by $\alpha\left(w_{i}\right)=w_{i+1}$ and $\alpha(0)=0$ (where $w \in\{a, b, c, d, e\}, i \in 2 n+1$ and addition in the subscript is taken modulo $2 n+1)$.

Let $\{s(1), \ldots, s(n)\}$ be some $n$-element subset of $S_{n}$. To apply Lemma 1.7 we need to show that the subsemigroup generated by $\{s(1), \ldots, s(n)\}$ is contained in $\mathcal{N}_{3} \cap \mathcal{J}$.

Let $L \subseteq 2 n+1$ denote the set of subscripts of the elements $s(1), \ldots, s(n)$. Evidently, there is some $i \leq n$ such that $i, i+1 \notin L$. Applying $n-i$ times the automorphism $\alpha$, we may assume that $i=2 n$ and $i+1=0$. So $\left\{s_{1}, \ldots, s_{n}\right\}$ is a subset of the set

$$
\{0\} \cup\left\{w_{i} \mid 0<i<2 n, w \in\{a, b, c, d, e\}\right\} \cup\left\{e_{2 n}\right\} .
$$

This set is a subuniverse of $\mathbf{S}_{n}$, and we denote the corresponding subsemigroup by $\mathbf{T}_{n}$. We now list some subsets of $\mathbf{T}_{n}$ that are easily verified as being strong:

$$
\begin{aligned}
& \left\{w_{i}\right\}, \text { where } w \in\{a, b, c, d\} \text { and } 0<i<2 n, \\
& \left\{e_{i}, e_{i+1}, \ldots, e_{2 n}\right\}, \text { where } i \leq 2 n .
\end{aligned}
$$

So $\mathbf{T}_{n}$ is $T_{0}$ with respect to $\mathcal{T}_{\mathbf{T}_{n}}$ and is obviously 3-nilpotent, whence lies in $\mathcal{J} \cap \mathcal{N}_{3}$, as required.

Lemma 1.7 now completes the proof that $\mathcal{J}$ is not finitely axiomatised.

\section{3. $\mathcal{O}$ IS NOT FINITELY AXIOMATISED}

We now turn our attention to the quasivariety $\mathcal{O}$ of all semigroups faithfully representable as semigroups of order preserving total maps on a chain. This class was 
shown to be nonfinitely axiomatised by Vernitskii [34, who also gave an example of a 3-nilpotent semigroup that is not contained in $\mathcal{O}$. Vernitskily's proof is itself not particularly difficult; however, in this section, we give a very easy proof of the result using only 3-nilpotent semigroups.

We first observe a nice structural characterisation of membership in $\mathcal{O}$ that can be found in Repnitskiǐ and Vernitskiǐ [22]. Recall that a preorder is a reflexive, transitive binary relation. A preorder $\leq$ on a set $S$ is total if $s \leq t$ or $t \leq s$ for any $s, t \in S$. A preorder $\leq$ on a semigroup $\mathbf{S}$ is right-compatible if the implication $a \leq b \rightarrow a c \leq b c$ holds for every $a, b, c \in S$.

Lemma 3.1 (Repnitskiǐ and Vernitskiǐ [22, Theorem 2.1]). A semigroup $\mathbf{S}$ is contained in $\mathcal{O}$ if and only if for every pair of elements $a, b \in S^{1}$ with $a \neq b$ there is $a$ right-compatible total preorder $\leq_{a, b}$ on $\mathbf{S}^{1}$ such that $a \mathbb{Z}_{a, b} b$ or $b \leq_{a, b} a$.

As in Section 2, we apply Lemma 1.7 with $\mathcal{J}=\mathcal{N}_{3} \cap \mathcal{O}$ and $\mathcal{Q}=\mathcal{O}$.

For each $n \in \mathbb{N}$ we define a semigroup $\mathbf{S}_{n}$ on the set

$$
\left\{a, b, c_{0}, \ldots, c_{2 n+2}, d_{0}, \ldots, d_{2 n+2}, 0\right\} .
$$

We let 0 be a multiplicative zero element and let the following table describe all of the nonzero products in $\mathbf{S}_{n}$ :

\begin{tabular}{c|cccccc}
$\cdot$ & $c_{0}$ & $c_{1}$ & $c_{2}$ & $\cdots$ & $c_{2 n+1}$ & $c_{2 n+2}$ \\
\hline$a$ & $d_{0}$ & $d_{1}$ & $d_{2}$ & $\cdots$ & $d_{2 n+1}$ & $d_{2 n+2}$ \\
$b$ & $d_{1}$ & $d_{2}$ & $d_{3}$ & $\cdots$ & $d_{2 n+2}$ & $d_{0}$
\end{tabular}

Let $\leq$ be a right-compatible total preorder on $\mathbf{S}_{n}^{1}$. We let $\equiv$ denote the relation $\leq \cap(\leq)^{-1}$. If $a \leq b$, then we have $d_{0}=a c_{0} \leq b c_{0}=d_{1}=a c_{1} \leq b c_{1}=d_{2}=$ $a c_{2} \leq \cdots=d_{2 n+1}=a c_{2 n+2} \leq b c_{2 n+2}=d_{0}$, showing $d_{0} \equiv \cdots \equiv d_{2 n+2}$. The same statements with the $\leq$ reversed show that $b \leq a$ implies $d_{0} \equiv \cdots \equiv d_{2 n+2}$ again. By Lemma 3.1, $\mathbf{S}_{n} \notin \mathcal{O}$.

Now observe that the map $\alpha: \mathbf{S}_{n} \rightarrow \mathbf{S}_{n}$ that fixes $a, b$ and 0 and increments the subscript of the other elements by 1 (modulo $2 n+3$ ) is an automorphism.

Let $s(1), \ldots, s(n)$ be some $n$-element subset of $S_{n}$. Evidently there is a number $i$ with $0 \leq i \leq 2 n+2$ such that none of the elements $s(j)$ have subscript equal to $i$ or $i+1$. By applying $\alpha$ sufficiently many times, we may assume $i=2 n+1$. Hence the subsemigroup generated by the elements $s(1), \ldots, s(n)$ is a subsemigroup of the following subsemigroup of $\mathbf{S}_{n}$, which we denote $\mathbf{T}_{n}$ (as before, the table defines the nonzero products):

\begin{tabular}{c|cccccc}
$\cdot$ & $c_{0}$ & $c_{1}$ & $c_{2}$ & $\cdots$ & $c_{2 n-1}$ & $c_{2 n}$ \\
\hline$a$ & $d_{0}$ & $d_{1}$ & $d_{2}$ & $\cdots$ & $d_{2 n-1}$ & $d_{2 n}$ \\
$b$ & $d_{1}$ & $d_{2}$ & $d_{3}$ & $\cdots$ & $d_{2 n}$ & $d_{2 n+1}$
\end{tabular}

Now we show, using Lemma 3.1, that $\mathbf{T}_{n}$ is representable by order preserving transformations of a chain. Define a preorder $\leq$ on $\mathbf{T}_{n}^{1}$ by the reflexive transitive closure of the following relation:

$$
1>a>b>c_{0}>\cdots>c_{2 n}>d_{0}>\cdots>d_{2 n+1}>0 .
$$

This is certainly a total preorder on $\mathbf{T}_{n}^{1}$. We leave it to the reader to check the easily verifiable fact that $\leq$ is right-compatible. By Lemma 3.1. we have shown that every $n$-generated subsemigroup of $\mathbf{S}_{n}$ is in $\mathcal{O}$, as required. 


\section{Part 2. Proofs of main results.}

\section{The MERGE PRODUCT OF 3-NILPOTENT SEMIGROUPS AND HOMOTOPY EMBEDDINGS}

In this section we present a method of translating the nonfinite basis properties of the particular semigroup $\mathbf{C}_{3,1}$ into any other proper 3-nilpotent semigroup. We also observe that a conjecture presented in 22 is false.

For any 3-nilpotent semigroup $\mathbf{S}$ we let $\mathbf{S}_{\mathrm{p}}:=\{x \in S \mid(\forall u, v) u v \neq x\}$ denote the prime elements of $\mathbf{S}$ and $\mathbf{S}_{\mathrm{c}}:=\{x \in S \backslash\{0\} \mid(\exists u, v) u v=x\}$ the nonzero composite elements.

Let $\mathbf{S}$ and $\mathbf{T}$ be two proper 3-nilpotent semigroups. We define a new semigroup $\mathbf{S} \bowtie \mathbf{T}$ (the merge product) to be the semigroup on the universe $S_{\mathrm{p}} \times T_{\mathrm{p}} \cup S_{\mathrm{c}} \times T_{\mathrm{c}} \cup\{0\}$ where 0 is a multiplicative zero element and where the other products are defined by

$$
(s, t) \cdot\left(s^{\prime}, t^{\prime}\right)= \begin{cases}\left(s s^{\prime}, t t^{\prime}\right) & \text { if } s s^{\prime} \in S_{\mathrm{c}} \text { and } t t^{\prime} \in T_{\mathrm{c}}, \\ 0 & \text { otherwise. }\end{cases}
$$

The merge product is clearly a 3-nilpotent semigroup.

Let $\mathbf{R}, \mathbf{S}$ and $\mathbf{T}$ be proper 3-nilpotent semigroups and $\phi: \mathbf{S} \rightarrow \mathbf{T}$ be a homomorphism. Fix some nonzero composite element $c \in R$ and define a map $\phi^{\rtimes}: \mathbf{R} \bowtie \mathbf{S} \rightarrow \mathbf{R} \bowtie \mathbf{T}$ by

$$
(r, s) \mapsto \begin{cases}(r, \phi(s)) & \text { if } r \in R_{\mathrm{p}} \text { and } \phi(s) \in T_{\mathrm{p}}, \\ (r, \phi(s)) & \text { if } r \in R_{\mathrm{c}} \text { and } \phi(s) \in T_{\mathrm{c}}, \\ (c, \phi(s)) & \text { if } r \in R_{\mathrm{p}} \text { and } \phi(s) \in T_{\mathrm{c}}, \\ 0 & \text { otherwise. }\end{cases}
$$

We now verify that this is a homomorphism. Consider $x, y \in R \bowtie S$ and suppose that $\phi^{\rtimes}(x y) \neq 0$. As $x y \neq 0$ we have $r_{1}, r_{2} \in R_{\mathrm{p}}, s_{1}, s_{2} \in S_{\mathrm{p}}$ with $r_{1} r_{2}=: r \in R_{\mathrm{c}}$ and $s_{1} s_{2}=: s \in S_{\mathrm{c}}$ and $x=\left(r_{1}, s_{1}\right)$ and $y=\left(r_{2}, s_{2}\right)$. Now, as $0 \neq \phi^{\rtimes}(x y)=\phi^{\rtimes}(r, s)$ we have $\phi(s) \neq 0$. As $r \in R_{\mathrm{c}}$, we have $\phi^{\rtimes}(r, s)=$ $(r, \phi(s))$. However, $\phi\left(s_{1}\right) \phi\left(s_{2}\right)=\phi(s)$, showing that $\phi\left(s_{1}\right), \phi\left(s_{2}\right) \in T_{\mathrm{p}}$ and then $\phi^{\rtimes}(x) \phi^{\rtimes}(y)=\left(r_{1}, \phi\left(s_{1}\right)\right)\left(r_{2}, \phi\left(s_{2}\right)\right)=\left(r, \phi\left(s_{1}\right) \phi\left(s_{2}\right)\right)=(r, s)=\phi^{\rtimes}(x y)$.

Almost identical arguments show that the assumption $\phi^{\rtimes}(x) \phi^{\rtimes}(y) \neq 0$ leads to $\phi^{\rtimes}(x y)=\phi^{\rtimes}(x) \phi^{\rtimes}(y)$, so that $\phi^{\rtimes}$ is a homomorphism.

Lemma 4.1. Let $\mathbf{S}$ and $\mathbf{T}$ be 3-nilpotent semigroups with $\mathbf{S} \in \mathbb{I S P}(\mathbf{T})$. For any 3-nilpotent semigroup $\mathbf{R}$ we have $\mathbf{R} \bowtie \mathbf{S} \in \operatorname{ISP}(\mathbf{R} \bowtie \mathbf{T})$.

Proof. Recall that the property $\mathbf{S} \in \mathbb{I S P}(\mathbf{T})$ is equivalent to the separation condition of Lemma 1.6 for every pair of distinct elements $a, b \in S$ we have a morphism $\phi: \mathbf{S} \rightarrow \mathbf{T}$ with $\phi(a) \neq \phi(b)$. One can use the morphisms $\phi^{\rtimes}: \mathbf{R} \bowtie \mathbf{S} \rightarrow \mathbf{R} \bowtie \mathbf{T}$ to witness the separation condition for $\mathbf{R} \bowtie \mathbf{S}$ relative to $\mathbf{R} \bowtie \mathbf{T}$.

Now we need to recall the notion of a homotopy of algebras in the sense of Sapir 24. A homotopy embedding of a semigroup $\mathbf{S}$ in a semigroup $\mathbf{T}$ is a triple of injective maps $\alpha: S \rightarrow T, \beta: S \rightarrow T$ and $\gamma: S \rightarrow T$ satisfying the homomorphismlike property $\alpha(g) \beta(h)=\gamma(g h)$. We mention in passing that when $\alpha, \beta, \gamma$ are bijections we have the notion of an isotopy, a fundamental tool in the study of objects related to Latin squares (see [5] for example) and in some universal algebraic contexts (see [33] for example). 
We will say that a universal sentence $\Psi$ (written in prenex form) is a split universal sentence if the variables appearing in it can be split into two disjoint sets $X$ and $Y$ such that each atomic formula in the sentence is of the form $x y \approx x^{\prime} y^{\prime}$ for some $x, x^{\prime} \in X$ and $y, y^{\prime} \in Y$. We say that $\Psi$ is a split quasi-identity if $\Psi$ is a split universal sentence and is a quasi-identity. The concept of a split quasi-identity was introduced by Sapir 24] who proved a fundamental connection between quasivarieties defined by split quasi-identities and homotopy embeddings. He showed 3 that a quasivariety $\mathcal{Q}$ of semigroups is definable by split quasi-identities if and only if every semigroup that is homotopy embeddable in a member of $\mathcal{Q}$ is itself in $\mathcal{Q}$.

Lemma 4.2. Let $\Phi$ be a split universal sentence. If $\mathbf{S}$ fails $\Phi$ and homotopy embeds in $\mathbf{T}$, then $\mathbf{T}$ fails $\Phi$.

Proof. Let $\alpha, \beta, \gamma: S \rightarrow T$ be the injective maps forming the homotopy embedding, and let $\theta$ be an assignment of variables into $S$ that witnesses a failure of $\Phi$. Let $X$ denote the variables appearing on the left of words in $\Phi$ and $Y$ denote those variables appearing on the right (so $X \cap Y=\varnothing$ ). The assignment $\bar{\theta}$ sending $x \in X$ to $\alpha(\theta(x))$ and $y \in Y$ to $\beta(\theta(y))$ witnesses a failure of $\Phi$ in $\mathbf{T}$.

Let $\mathcal{O}_{\text {mon }}$ denote the quasivariety of monoids from $\mathcal{O}$ considered in the type $\langle 2,0\rangle$. In 22 a basis for the quasi-identities of $\mathcal{O}_{\text {mon }}$ is given and it is observed that these are split quasi-identities. Following an analogy with the semigroup case, Repnitskir and Vernitskii 22 conjectured that there is a characterisation of $\mathcal{O}_{\text {mon }}$ in terms of forbidden homotopy embeddings (in the sense described above for semigroups). More precisely, they conjecture that a finite monoid $\mathbf{M}$ is in $\mathcal{O}_{\text {mon }}$ if and only if the only group that is homotopy embedded in $\mathbf{M}$ is the trivial group. We now observe that the conjecture is false, although the failure to homotopy embed any nontrivial group is a necessary condition for membership in $\mathcal{O}$ (whence also for $\mathcal{O}_{\text {mon }}$ ).

It is not hard to see that the only finite group in $\mathcal{O}$ is the one-element group. This can be proved directly from the definition of $\mathcal{O}$ or using Lemma 3.1 let $\mathbf{G}$ be a finite group with an element $a \neq 1$. If $a \leq 1$ under some right-compatible preorder, then $a^{n} \leq a^{n-1}$ for all $n$. Let $d$ be the order of $a$. We have $a \geq a^{2} \geq \cdots \geq a^{d}=1$ so that $a \equiv 1$. A similar argument shows that if $a \geq 1$, then $a \equiv 1$. By Lemma 3.1 we have $\mathbf{G} \notin \mathcal{O}$. By Lemma 4.2, no member of $\mathcal{O}$ can homotopy embed a nontrivial group. (Note however that the additive group of integers is contained in $\mathcal{O}$.)

To see why the condition is not a sufficient condition for membership in $\mathcal{O}_{\text {mon }}$, observe that if there is a homotopy embedding of a group in some finite monoid $\mathbf{T}$, then the table of $\mathbf{T}$ must contain an $m \times m$ Latin square for some $m>1$. The semigroups $\mathbf{S}_{n}$ in the proof in Section 3 are not contained in $\mathcal{O}$, whence neither are the monoids $\mathbf{S}_{n}^{1}$ obtained by adjoining an identity element. However there are no nontrivial Latin squares in the table of $\mathbf{S}_{n}^{1}$.

The following result will be key in our translation of the INFQB properties of $\mathbf{C}_{3,1}$ to other 3 -nilpotent semigroups.

Lemma 4.3. For any two proper 3-nilpotent semigroups $\mathbf{R}$ and $\mathbf{S}$, there is a homotopy embedding of $\mathbf{S}$ into $\mathbf{R} \bowtie \mathbf{S}$.

\footnotetext{
${ }^{3}$ In fact Sapir concentrates on prevarieties-or ISP-closed classes-where one is allowed an infinite conjunction in the premise of a quasi-identity.
} 
Proof. We use only that $\mathbf{R}$ is proper. Consider some nonzero product $a b=c \neq 0$ in $\mathbf{R}$. Define the following injective maps $\alpha, \beta, \gamma$ from $S$ into $R \bowtie S$ :

$$
\alpha(s)=\gamma(s)=\left\{\begin{array}{ll}
(a, s) & \text { if } s \in S_{p}, \\
(c, s) & \text { if } s \in S_{c}, \\
0 & \text { if } s=0,
\end{array} \quad \beta(s)= \begin{cases}(b, s) & \text { if } s \in S_{p} \\
(c, s) & \text { if } s \in S_{c} \\
0 & \text { if } s=0 .\end{cases}\right.
$$

It is easy to see that these are indeed injective and satisfy $\alpha(a) \beta(b)=\gamma(a b)$.

Lemma 4.4. Let $\Phi$ be a split universal sentence failing on a 3-nilpotent semigroup $\mathbf{S}$. Then for any other 3-nilpotent semigroup $\mathbf{R}$ we have that $\Phi$ fails on $\mathbf{R} \bowtie \mathbf{S}$.

Proof. This follows immediately from Lemma 4.3 and Lemma 4.2.

Lemma 4.5. Let $\mathbf{R}$ and $\mathbf{S}$ be proper 3-nilpotent semigroups with $\mathbf{R}$ finite. Let $Q$ be a universal class containing the quasivarieties $\mathbb{Q}(\mathbf{R})$ and $\mathbb{Q}(\mathbf{R} \bowtie \mathbf{S})$. If $\mathcal{Q}$ satisfies a system of split quasi-identities $\Sigma$ such that $\Sigma$ forms a nonfinitely axiomatisable system in the quasi-equational theory of $\mathbf{R}$, then $\Sigma$ forms a nonfinitely axiomatisable system in the quasi-equational theory of $\mathbf{R} \bowtie \mathbf{S}$.

Proof. As $\Sigma$ is not finitely axiomatisable in the quasi-equational theory of $\mathbf{R}$, there are for each $n \in \mathbb{N}$, semigroups $\mathbf{R}_{n}$ whose $n$-generated subsemigroups lie in $\mathbb{Q}(\mathbf{R})$ but which fail some member $\Phi_{n}$ of $\Sigma$. Now let $\mathbf{S}_{n}:=\mathbf{R} \bowtie \mathbf{R}_{n}$. By Lemma 4.4. we have that $\mathbf{S}_{n} \not \models \Phi_{n}$, showing that $\mathbf{S}_{n} \notin \mathcal{Q} \supseteq \mathbb{Q}(\mathbf{R} \bowtie \mathbf{S})$. However every $n$-generated subsemigroup $\mathbf{S}_{n}^{\prime}$ of $\mathbf{S}_{n}$ is a subsemigroup of $\mathbf{R} \bowtie \mathbf{R}_{n}^{\prime}$ for some $n$ generated subsemigroup $\mathbf{R}_{n}^{\prime}$ of $\mathbf{R}_{n}$. Now $\mathbf{R}_{n}^{\prime} \in \mathbb{I} \mathbb{S P}(\mathbf{R})$ by assumption, so by Lemma 4.1 we have $\mathbf{R} \bowtie \mathbf{S}_{n}^{\prime} \in \mathbb{I S P}(\mathbf{R} \bowtie \mathbf{S})$.

The reader will readily verify that the semigroup $\mathbf{C}_{3,1}$ acts as an identity element for the merge product. In other words, we have $\mathbf{C}_{3,1} \bowtie \mathbf{S} \cong \mathbf{S}$ for any proper 3-nilpotent semigroup $\mathbf{S}$. We will use this property to translate properties of $\mathbf{C}_{3,1}$ into arbitrary proper 3-nilpotent semigroups; the method is encapsulated by Lemma 5.7 below.

\section{Monogenic Semigroups}

We now examine some constructions that are defined for any numbers $i, p \in \mathbb{N}$ and any alphabet $X$. In fact we could get by using only the cases $i=3,4$; however, it is easier to give the general case than consider these separately. The construction is a generalisation of the method used by Clark, Davey, Jackson and Pitkethly 2 where it was used to show that the semigroups $\mathbf{C}_{i, p}$ are not finitely q-based if and only if $i \geq 3$.

Let $\mathbf{C}(Z)$ denote the free commutative semigroup freely generated by the set $Z$. Elements of $Z$ will be referred to as letters and elements of $C(Z)$ will be referred to as words. The length of a word $w$ is the number of letters (including repetitions) required to form $w$ as a product in $\mathbf{C}(Z)$. We let $|w|$ denote the length of $w$ and $c(w)$ (the content of $w$ ) denote the set of letters appearing in $w$. For example, the words $a b b a c$ and $a b a c b$ coincide, have length 5 and have content $\{a, b, c\}$. Recall the numerical equivalence relation $\theta_{i, p}$ defined in Subsection 1.1. Let us define a congruence $\rho_{i, p}$ on $\mathbf{C}(Z)$ by letting $u \rho v$ if one of the following holds:

(1) $|u|,|v| \geq i$ and $|u| \equiv|v| \bmod p$ (that is, $|u| \equiv|v| \bmod \theta_{i, p}$ );

(2) $|u|=|v|<i$ and $c(u)=c(v)$. 
It is routine to see that this is a congruence; we let $\mathbf{F}_{i, p}(Z):=\mathbf{C}(Z) / \rho_{i, p}$. We adjust the definitions of length and content for elements of $C(Z)$ to elements of $F_{i, p}(Z)$ as follows: for $w \in C(Z)$, the length $\left|w / \rho_{i, p}\right|$ of $w / \rho_{i, p}$ is defined modulo $\theta_{i, p}$ as $|w|$; the content $c\left(w / \rho_{i, p}\right)$ is only defined when $|w| \leq i-1$, in which case $c\left(w / \rho_{i, p}\right):=c(w)$. These definitions are easily seen to be unambiguous. It will also do no harm to refer to elements of $\mathbf{F}_{i, p}(Z)$ as words in $Z$. (Of course, words that are distinct in $\mathbf{C}(Z)$ may be identical in $\mathbf{F}_{i, p}(Z)$ : $a a b$ coincides with $a b b$ in $\mathbf{F}_{4,1}(\{a, b\})$ for example.)

Let us say that an equivalence relation $\theta$ on $F_{i, p}(Z)$ is clean if whenever $u, v$ are distinct $\theta$-related words in $F_{i, p}(Z)$, then $|u|=|v|=i-1$. The definition of $\mathbf{F}_{i, p}(Z)$ ensures that every clean equivalence is a congruence. When $\theta$ is a clean congruence, we write $\mathbf{G}_{i, p}(Z, \theta)$ in place of $\mathbf{F}_{i, p}(Z) / \theta$. Since a clean congruence $\theta$ preserves word length modulo $\theta_{i, p}$, we may continue to unambiguously write $|u|$ to denote the length of an element $u \in \mathbf{G}_{i, p}(Z, \theta)$ modulo $\theta_{i, p}$. For words $w$ in $\mathbf{F}_{i, p}(Z)$ with $|w|<i-1$, we have $w / \theta=\{w\}$, and in such cases we occasionally abuse notation and use $w$ to denote the element $w / \theta$. This convention means that we can again consider $Z$ to be the set of generators of $\mathbf{G}_{i, p}(Z, \theta)$ (instead of $\{z / \theta \mid z \in Z\}$ ).

We observe that if $Y \subseteq Z$, then the subsemigroup of $\mathbf{G}_{i, p}(Z, \theta)$ generated by $Y$ is precisely $\mathbf{G}_{i, p}\left(Y, \theta \uparrow_{\mathbf{F}_{i, p}(Y)}\right)$, where $\theta \uparrow_{\mathbf{F}_{i, p}(Y)}$ is the restriction of $\theta$ to $\mathbf{F}_{i, p}(Y)$. For notational brevity, we write $\mathbf{G}_{i, p}(Y, \theta)$ in place of $\mathbf{G}_{i, p}\left(Y, \theta \uparrow_{\mathbf{F}_{i, p}(Y)}\right)$. Also, we mention that there is a unique maximal subgroup $\mathbf{G}_{p}$ of $\mathbf{G}_{i, p}(Z, \theta)$ which is a cyclic group $\mathbf{Q}_{p}$ of order $p$ whose elements are $q_{0}, \ldots, q_{p-1}$, where $q_{j}$ denotes the set of all words $u$ of $\mathbf{C}(Z)$ with length equal to $j+i$ modulo $\theta_{i, p}$.

We need some further definitions. First recall that if $\mathbf{M}$ is a semigroup and $S \subseteq M$, then $\operatorname{Sg}^{\mathbf{M}}(S)$ denotes the subsemigroup generated by $S$. We use $\langle S\rangle$ when the choice of $\mathbf{M}$ is clear. Let us say that a subset $S \subseteq Z$ is stable (in $\mathbf{G}_{i, p}(Z, \theta)$ ) when the following property holds: if $a_{1}, \ldots, a_{k} \in S, b_{1}, \ldots, b_{\ell} \in Z$ and $a_{1} \ldots a_{k}=b_{1} \ldots b_{\ell} \notin Q_{p}$ then $b_{1}, \ldots, b_{\ell} \in S$. (The definition of a clean congruence ensures the only nontrivial instances of this property will be when $k=\ell=i-1$.) We also define an operator $D_{\mathbf{G}_{i, p}}$ on subsets of $G_{i, p}(Z, \theta) \backslash Q_{p}$ by

$$
D_{\mathbf{G}_{i, p}}: A \mapsto\{a \in Z \mid a \in A \text { or }(\exists b) a b \in A\} .
$$

In other words, $D_{\mathbf{G}_{i, p}}(A)$ gives the set of all generators $a \in Z$ that divide some element of $A$. The reader can easily verify that a set $S \subseteq Z$ is stable if and only if $D_{\mathbf{G}_{i, p}}\left(\langle S\rangle \backslash Q_{p}\right)=S$. When there is no confusion as to which semigroup we are working in, we drop the subscript $\mathbf{G}_{i, p}$ from $D_{\mathbf{G}_{i, p}}$.

Lemma 5.1. Fix an alphabet $Z$ and let $\mathbf{F}$ abbreviate $\mathbf{F}_{i, p}(Z)$. A subset $S \subseteq Z$ is stable in $\mathbf{B}:=\mathbf{G}_{i, p}(Z, \theta)$ if and only if whenever $u, v$ are distinct $\theta$-related elements of $\mathbf{F}$, then

$$
u \in \operatorname{Sg}^{\mathbf{F}}(S) \Rightarrow D_{\mathbf{F}}(v) \subseteq S .
$$

Proof. The "only if" follows immediately from the definition of stable. Now say that $S$ is not stable in B. So there are elements $a_{1}, \ldots, a_{k} \in S \subseteq Z$ and $b_{1}, \ldots, b_{\ell} \in Z$ with $a_{1} \ldots a_{k}=b_{1} \ldots b_{\ell} \notin Q_{p}$ and with some $b_{j} \notin S$. In particular, $\left\{a_{1}, \ldots, a_{k}\right\} \neq$ $\left\{b_{1}, \ldots, b_{\ell}\right\}$, so the words $u:=a_{1} \ldots a_{k}$ and $v:=b_{1} \ldots b_{\ell}$ are distinct in $\mathbf{F}_{i, p}(Z)$. Hence we must have $u \theta v$. Clearly $u \in \operatorname{Sg}^{\mathbf{F}}(S)$ but $D_{\mathbf{F}}(v) \nsubseteq S$. 
Every clean congruence $\theta$ encountered in this article has the following property:

(*) $\quad$ if $u$ and $v$ are distinct words with $u \theta v$, then $|c(u)|=|c(v)|=i-1$.

Property (荬) makes verification of the stability of certain sets easier. The idea is encapsulated in the following lemma.

Lemma 5.2. Let $\mathbf{F}$ abbreviate $\mathbf{F}_{i, p}(Z)$ and consider a clean congruence $\theta$ satisfying property (因). If $w \in F \backslash Q$ has $w / \theta$ a singleton class, then $c(w)$ is stable in $\mathbf{G}_{i, p}(Z, \theta)$.

Proof. Put $S:=c(w)$ and consider distinct $\theta$-related words $u, v$. Every length $i-1$ word in $\operatorname{Sg}^{\mathbf{F}}(S)$ either has a repeated letter or is equal to $w$. By property (荬), $u \notin \operatorname{Sg}^{\mathbf{F}}(S)$, and Lemma 5.1 ensures that $S$ is stable.

The following elementary fact generalises a method from [2].

Lemma 5.3. If $S \subseteq Z$ is a stable set in some $\mathbf{B}:=\mathbf{G}_{i, p}(Z, \theta)$, then the map $\nu_{S}: \mathbf{B} \rightarrow \mathbf{C}_{i, p}$ defined by

$$
z \mapsto \begin{cases}a^{|z|} & \text { if } z \in\langle S\rangle, \\ a^{i p+|z|} & \text { otherwise }\end{cases}
$$

is a homomorphism.

Proof. Throughout this proof, we let $u, v$ be elements of $B$.

First we show that

$$
u \cdot v \in\langle S\rangle \backslash Q_{p} \text { if and only if } u, v \in\langle S\rangle \backslash Q_{p} \text { and }|u|+|v|<i \text {. }
$$

The "if" part of (头) is trivial. Now say that $u \cdot v \in\langle S\rangle \backslash Q_{p}$. Then $D_{\mathbf{B}}(\{u, v\}) \subseteq$ $D_{\mathbf{B}}(u \cdot v) \subseteq D_{\mathbf{B}}\left(\langle S\rangle \backslash Q_{p}\right)=S$, so that $u, v \in\langle S\rangle$. Since $|u|+|v|=|u \cdot v|<i$, we have $u, v \in\langle S\rangle \backslash Q_{p}$. So (买) holds.

If $|u|+|v| \geq i$, then $|u|+|v| \equiv i p+|u|+|v| \bmod \theta_{i, p}$, and so we have $\nu_{S}(u \cdot v)=$ $a^{|u|+|v|}=a^{i p+|u|+|v|}$, showing $\nu_{S}(u) \cdot \nu_{S}(v)=\nu_{S}(u \cdot v)$. Now say that $|u|+|v|<i$.

If $u \cdot v \in\langle S\rangle$, then by (函) we have $\nu_{S}(u) \cdot \nu_{S}(v)=a^{|u|} a^{|v|}=a^{|u|+|v|}=a^{|u \cdot v|}=$ $\nu_{S}(u \cdot v)$. If $u \cdot v \notin\langle S\rangle$, then at least one of $u$ or $v$ - say $u$-is not in $\langle S\rangle$, and

$$
\nu_{S}(u) \nu_{S}(v)=\left\{\begin{array}{l}
a^{i p+|u|} a^{|v|} \text { or } \\
a^{i p+|u|} a^{i p+|v|}
\end{array}\right\}=a^{i p+|u|+|v|}=\nu_{S}(u \cdot v),
$$

as required.

We refer to the map $\nu_{S}$ as the natural map of the stable set $S$.

We now concentrate on the particular case $\mathbf{C}_{3,1}$. Let us consider any semigroup $\mathbf{G}_{3,1}(X, \theta)$, and let $i>2, p \geq 1$ be arbitrary integers. We construct a new semigroup $\mathbf{H}_{i, p}(X, \theta)$, which will be equal to $\mathbf{G}_{i, p}\left(X^{\sharp}, \theta^{\sharp}\right)$ for some set $X^{\sharp}$ and clean congruence $\theta^{\sharp}$. We let $X^{\sharp}:=\left\{a_{x, j} \mid 1 \leq j \leq i-2, x \in X\right\} \cup\left\{b_{x} \mid x \in X\right\}$ and define the clean congruence $\theta^{\sharp}$ on $\mathbf{F}_{i, p}\left(X^{\sharp}\right)$ by identifying $a_{x_{1}, 1} \ldots a_{x_{1}, i-2} b_{y_{1}}$ with $a_{x_{2}, 1} \ldots a_{x_{2}, i-2} b_{y_{2}}$ whenever $x_{1} y_{1} \theta x_{2} y_{2}$. Observe that $\theta^{\sharp}$ always satisfies property (函), even if $\theta$ fails it. Define $\mathbf{H}_{i, p}(X, \theta):=\mathbf{G}_{i, p}\left(X^{\sharp}, \theta^{\sharp}\right)$.

Lemma 5.4. $\mathbf{H}_{i, p}(X, \theta) \in \mathbb{I S P}\left(\mathbf{C}_{i, p}\right)$ if and only if $\mathbf{G}_{3,1}(X, \theta) \in \mathbb{I S P}\left(\mathbf{C}_{3,1}\right)$. 
Proof. Suppose that $\mathbf{G}_{3,1}(X, \theta) \in \mathbb{I S P}\left(\mathbf{C}_{3,1}\right)$. Let $x, y$ be distinct elements of $\mathbf{H}_{i, p}(X, \theta)$. For the remainder of the proof, we abbreviate $\mathbf{F}_{i, p}\left(X^{\sharp}, \theta^{\sharp}\right), \mathbf{G}_{3,1}(X, \theta)$ and $\mathbf{H}_{i, p}(X, \theta)$ by $\mathbf{F}, \mathbf{G}$ and $\mathbf{H}$ respectively.

If one of $x$ or $y$ is a group element, then the map $\nu_{X^{\sharp}}$ separates $x$ and $y$. Now assume that neither $x$ nor $y$ is a group element.

Case 1 . One of $x$ or $y$ is a singleton congruence class of $\theta^{\sharp}$.

In this case we may assume without loss of generality that $x$ is a singleton congruence class. Also, if $y$ is a singleton congruence class, then we can assume that either $|x| \neq|y|$ or if $|x|=|y|$, then $D_{\mathbf{H}}(y) \nsubseteq D_{\mathbf{H}}(x)$. By Lemma 5.2, we have that $S:=D_{\mathbf{H}}(x)$ is stable, and then $\nu_{S}$ separates $x$ and $y$.

Case 2. Both $x$ and $y$ are nonsingleton congruence classes of $\theta^{\sharp}$.

Suppose that $x=a_{x_{1}, 1} \ldots a_{x_{1}, i-2} b_{y_{1}} / \theta^{\sharp}$ and $y=a_{x_{2}, 1} \ldots a_{x_{2}, i-2} b_{y_{2}} / \theta^{\sharp}$. Now let $\phi: \mathbf{G} \rightarrow \mathbf{C}_{3,1}$ be a map separating the elements $x_{1} y_{1} / \theta$ and $x_{2} y_{2} / \theta$ of $\mathbf{G}$. It is clear that $\phi$ sends one of these elements to the zero element of $\mathbf{C}_{3,1}$ and the other to the element $a^{2}$; say $\phi\left(x_{2} y_{2}\right)=0$, so that at least one of $\phi\left(x_{2}\right)$ and $\phi\left(y_{2}\right)$ is not equal to $a$. Define a subset $S$ of $X^{\sharp}$ by $S:=\left\{a_{z, j}, b_{z} \mid z \in X\right.$ has $\left.\phi(z)=a\right\}$. We use Lemma 5.1 to show that $S$ is stable.

Say $u$ and $v$ are distinct elements of $\mathbf{F}$ with $u \theta^{\sharp} v$ and $u \in \operatorname{Sg}^{\mathbf{F}}(S)$. So $u=a_{x_{3}, 1} \ldots a_{x_{3}, i-2} b_{y_{3}}$ and $v=a_{x_{4}, 1} \ldots a_{x_{4}, i-2} b_{y_{4}}$, where $x_{3} y_{3} \theta x_{4} y_{4}$ in $\mathbf{F}_{3,1}(X)$. Also, the assumption $u \in \operatorname{Sg}^{\mathbf{F}}(S)$ implies that $\phi\left(x_{3}\right)=\phi\left(x_{4}\right)=a$. Since $\phi$ is a homomorphism from $\mathbf{G}$ and the products $x_{3} \cdot y_{3}$ and $x_{4} \cdot y_{4}$ are equal in $\mathbf{G}$ we have $\phi\left(x_{4} y_{4}\right)=\phi\left(x_{3} y_{3}\right)=a^{2}$. So $\phi\left(x_{4}\right)=\phi\left(y_{4}\right)=a$. From this it easily follows that $D_{\mathbf{F}}(v) \subseteq S$, as required. So by Lemma 5.1, $S$ is stable.

Now we must show that $\nu_{S}$ separates $x$ and $y$. For this it suffices to show that $D_{\mathbf{F}}(y) \not \subset S$. But this follows because of the definition of $S$ and the fact that one of $\phi\left(x_{2}\right)$ and $\phi\left(y_{2}\right)$ is not equal to $a$. This completes the proof of Case 2, and also that $\mathbf{H} \in \mathbb{I S P}\left(\mathbf{C}_{i, p}\right)$.

Now we show the reverse direction. Say that $\mathbf{H} \in \mathbb{I S P}\left(\mathbf{C}_{i, p}\right)$. For each distinct $x$ and $y$ in $\mathbf{G}$ we want to find a homomorphism $\phi: \mathbf{G} \rightarrow \mathbf{C}_{3,1}$ with $\phi(x) \neq \phi(y)$. We observe that this is very easy if $|x|=1$ or $|y|=1$, or if one of $x$ or $y$ is the single group element (the multiplicative zero element). So we are left with the case that $|x|=|y|=2$. In this case, there are $x_{1}, y_{1}, x_{2}, y_{2} \in X$ with $x_{1} y_{1}=x$ and $x_{2} y_{2}=y$. Now consider the elements $\bar{x}:=a_{x_{1}, 1} \ldots a_{x_{1}, i-2} b_{y_{1}}$ and $\bar{y}:=a_{x_{2}, 1} \ldots a_{x_{2}, i-2} b_{y_{2}}$ of $\mathbf{H}$, which are distinct because $x$ and $y$ are distinct. Hence there exists a homomorphism $\psi: \mathbf{H} \rightarrow \mathbf{C}_{i, p}$ with $\psi(\bar{x}) \neq \psi(\bar{y})$. Now if both $\psi(\bar{x})$ and $\psi(\bar{y})$ are group elements, then we have $\psi(\bar{x})=\psi(\bar{x})^{p}=\psi\left((\bar{x})^{p}\right)=\psi\left((\bar{y})^{p}\right)=\psi(\bar{y})^{p}=\psi(\bar{y})$, a contradiction. So we can assume without loss of generality that $\psi(\bar{x})$ is not a group element. Since both $\bar{x}$ and $\bar{y}$ are products of length $i-1$, and since there is only one nongroup element with this property in $\mathbf{C}_{i, p}$, we must have $\psi(\bar{x})=a^{i-1}$ and that $\psi(\bar{y})$ is a group element. Let $S \subseteq X$ be the set of all letters $x^{\prime}$ for which either $\psi\left(a_{x^{\prime}, 1}\right)=$ $\cdots=\psi\left(a_{x^{\prime}, i-2}\right)=a$ or $\psi\left(b_{x^{\prime}}\right)=a$. Now $S$ is nonempty because we have $x_{1}, y_{1} \in$ $S$. However it is very easy to use Lemma 5.1 to show that $S$ is stable and that $y \notin \operatorname{Sg}^{\mathbf{F}_{3,1}(X)}(S)$. Hence the natural map of $S$ separates $x$ and $y$, as required.

Let $\Phi:=\&_{1 \leq i \leq n} p_{i} \approx q_{i} \rightarrow p_{0} \approx q_{0}$ be a split quasi-identity in some variables $x_{1}, \ldots, x_{m}$. Let $\mathbf{S}$ be a finite semigroup with minimal ideal $I$. We say that $\Phi$ fails in $\mathbf{S} \backslash I$ if there is an interpretation $\phi:\left\{x_{1}, \ldots, x_{m}\right\}^{+} \rightarrow \mathbf{S}$ such that $\Phi$ fails under $\phi$ and $\left\{p_{0}, q_{0}, p_{1}, q_{1}, \ldots, q_{n}\right\} \subseteq \phi^{-1}(S \backslash I)$. 
Lemma 5.5. Let $\Phi$ be a split quasi-identity. If $\Phi$ fails in $\mathbf{G}_{3,1}(X, \theta) \backslash\{0\}$, then $\Phi$ fails in $\mathbf{H}_{i, p}(X, \theta) \backslash Q_{p}$ (and in particular, in $\mathbf{H}_{i, p}(X, \theta)$ ).

Proof. Say the variables of $\Phi$ are $x_{1}, \ldots, x_{k}, y_{1}, \ldots, y_{\ell}$, where the $x_{j}$ are the letters appearing in the first position in words of $\Phi$, while the $y_{j}$ are the letters appearing in the second position. If $\phi$ is the failing assignment into $\mathbf{G}_{3,1}(X, \theta) \backslash\{0\}$ we must have $\phi\left(\left\{x_{1}, \ldots, x_{k}, y_{1}, \ldots, y_{\ell}\right\}\right) \subseteq Z$. Now define

$$
\phi^{\sharp}:\left\{x_{1}, \ldots, x_{k}, y_{1}, \ldots, y_{\ell}\right\}^{+} \rightarrow \mathbf{H}_{i, p}(X, \theta)
$$

by $\phi^{\sharp}\left(x_{j}\right)=a_{x_{j}, 1} \ldots a_{x_{j}, i-2}$ and $\phi^{\sharp}\left(y_{j}\right)=b_{y_{j}}$. It is clear that $\phi^{\sharp}$ causes $\Phi$ to fail in $\mathbf{H}_{i, p}(X, \theta) \backslash Q_{p}$.

We could alternatively prove this lemma by observing that there is a homotopy embedding of the partial semigroup $\mathbf{G}_{3,1}(X, \theta) \backslash\{0\}$ into the partial semigroup on $\mathbf{H}_{i, p}(X, \theta) \backslash Q_{p}$.

Lemma 5.6. Say that $\ell:=n(i-1)+1$. Then every n-generated subsemigroup of $\mathbf{H}_{i, p}(X, \theta)$ embeds into $\mathbf{H}_{i, p}(Y, \theta)$ for some $Y \subseteq X$ with $|Y|<\ell$.

Proof. Let $A$ be some $n$-element subset of $\mathbf{H}(X, \theta)$, and let $B$ denote the nongroup elements of $A$. Each element $a \in B$ either has $\left|D_{\mathbf{G}_{i, p}\left(X^{\sharp}, \theta^{\sharp}\right)}(a)\right| \leq i-1$ or is of the form $a_{x, 1} \ldots a_{x, i-2} b_{y} / \theta^{\sharp}$ for some $x, y \in X$. For each $a \in B$ pick an element $a^{\prime}$ from $\mathbf{F}_{i, p}\left(X^{\sharp}\right)$ with $a=a^{\prime} / \theta^{\sharp}$. So for each $a \in B$ we have $\left|D_{\mathbf{F}_{i, p}\left(X^{\sharp}\right)}\left(a^{\prime}\right)\right| \leq i-1$, showing that the union

$$
B^{\prime}:=\bigcup_{a \in B} D_{\mathbf{F}_{i, p}\left(X^{\sharp}\right)}\left(a^{\prime}\right)
$$

has size at most $n(i-1)$. Let $Y$ be the subset of $X$ consisting of all letters appearing as the first subscript of some member of $B^{\prime}$; so $|Y| \leq n(i-1)<\ell$. Each $a \in A$ belongs to the subsemigroup $\mathbf{H}_{i, p}(Y, \theta)$ of $\mathbf{H}_{i, p}(X, \theta)$ as a product of elements from $Y^{\sharp}$. So $A \subseteq \mathbf{H}_{i, p}(Y, \theta)$, as required.

We now summarise the results of this section and the last in the form of the following refinement of Lemma 1.7 (and which will be the template for the proofs of Theorem $1.1_{\text {i) }}$ and Theorem $\left.1.11_{\text {(ii) } \vee(\text { iii })}\right)$.

Lemma 5.7. Let $Q$ be a quasivariety. Say that there is a set of split quasi-identities $\Sigma$ with the following properties:

(1) $\mathcal{Q}=\Sigma$;

(2) for each $m \in \mathbb{N}$ there is a semigroup $\mathbf{T}_{m}$ of the form $\mathbf{G}_{3,1}(Z, \theta)$ for some $Z, \theta$ such that

(a) some member of $\Sigma$ fails in $\mathbf{T}_{m} \backslash\{0\}$;

(b) every m-generated subsemigroup of $\mathbf{T}_{m}$ is contained in $\operatorname{ISP}\left(\mathbf{C}_{3,1}\right)$.

Every universal class $\mathcal{U} \subseteq \mathcal{Q}$ containing the quasivariety of a proper 3-nilpotent semigroup, or of a finite semigroup with index more than 2 , has no finite axiomatisation in first order logic.

Proof. First say that $\mathcal{U}$ contains the quasivariety of a proper 3-nilpotent semigroup S. We apply Lemma 1.7 with $\mathcal{J}:=\mathbb{I S P}(\mathbf{S}), \mathcal{K}:=\mathcal{U}$ and $\mathbf{S}_{n}:=\mathbf{S} \bowtie \mathbf{T}_{n}$. By Lemma 4.4 and condition 2(a) we have $\mathbf{S}_{n} \not \models \Sigma$ and hence is not contained in $Q$. However every $n$-generated subsemigroup of $\mathbf{S}_{n}$ is a subsemigroup of $\mathbf{S} \bowtie \mathbf{R}_{n}$ for some $n$-generated subsemigroup $\mathbf{R}_{n}$ of $\mathbf{T}_{n}$. Property 2(b) and Lemma 4.1 together show that $\mathbf{S} \bowtie \mathbf{R}_{n} \in \mathbb{Q}\left(\mathbf{S} \bowtie \mathbf{C}_{3,1}\right)=\mathbb{Q}(\mathbf{S})$ (since $\mathbf{S} \bowtie \mathbf{C}_{3,1} \cong \mathbf{S}$ ). Hence every 
$n$-generated subsemigroup of $\mathbf{S}_{n}$ is contained in $\mathbb{Q}(\mathbf{S})$ and Lemma 1.7 applies. This completes the proof in the case that $\mathcal{U}$ contains the quasivariety of some proper 3 -nilpotent semigroup.

Now say that $\mathbf{T}$ is a semigroup of finite index more than 2 and $U \supseteq \mathbb{Q}(\mathbf{T})$. So there is a monogenic subsemigroup $\mathbf{C}_{i, p}$ of $\mathbf{T}$ with index more than 2 , and $\mathcal{U} \supseteq \mathbb{Q}\left(\mathbf{C}_{i, p}\right)$.

We apply Lemma 1.7 with $\mathcal{J}:=\mathbb{I S P}\left(\mathbf{C}_{i, p}\right), \mathcal{K}:=\mathcal{U}$.

Now for each $n$, the semigroup $\mathbf{T}_{n}$ is of the form $\mathbf{G}_{3,1}\left(X_{n}, \theta_{n}\right)$ for some set $X_{n}$ and clean congruence $\theta_{n}$. Put $\ell:=n(i-1)+1$ and choose $\mathbf{S}_{n}:=\mathbf{H}_{i, p}\left(X_{\ell}, \theta_{\ell}\right)$. By Lemma 5.5 and assumption 2(a) of the current lemma we have $\mathbf{S}_{n} \not \models \Sigma$, showing $\mathbf{S}_{n} \notin \mathcal{Q}$. Now consider an $n$-generated subsemigroup $\mathbf{S}$ of $\mathbf{S}_{n}$. By Lemma [5.6, there is an $(\ell-1)$-element subset $Y \subseteq X_{\ell}$ such that $\mathbf{S}$ is a subsemigroup of $\mathbf{H}_{i, p}\left(Y, \theta_{\ell}\right)$. Now $\mathbf{G}_{3,1}\left(Y, \theta_{\ell}\right)$ is an $(\ell-1)$-generated subsemigroup of $\mathbf{G}_{3,1}\left(X_{\ell}, \theta_{\ell}\right)=\mathbf{T}_{\ell}$, and so property 2(b) shows that $\mathbf{G}_{3,1}\left(Y, \theta_{\ell}\right) \in \mathbb{I S P}\left(\mathbf{C}_{3,1}\right)$. By Lemma 5.4 we have $\mathbf{S} \leq \mathbf{H}_{i, p}\left(Y, \theta_{\ell}\right) \in \mathbb{I S P}\left(\mathbf{C}_{i, p}\right)$. The result now follows from Lemma 1.7.

\section{Proof of Theorem $1.1_{\text {I })}$}

In this section we prove Theorem $1.1_{\mathrm{i}}$, using Lemma 5.7 and the constructions of Section 5 ,

The set of split quasi-identities $\Sigma$ required for Lemma 5.7 will be $\left\{\Phi_{n} \mid n \in \mathbb{N}\right\}$, where $\Phi_{n}$ denotes the following split quasi-identity:

$$
\left(\underset{0 \leq j \leq n}{\&} x_{j} v_{j} \approx u_{j} v_{j} \approx u_{j} y_{j} \approx x_{j-1} y_{j-1}\right) \rightarrow x_{0} y_{0} \approx x_{1} y_{1} .
$$

This quasi-identity is modelled on the nonzero products in the semigroup $\mathbf{S}_{n}$ of Section 2. The proof that $\mathcal{J}$ satisfies $\Phi_{n}$ is essentially the same as the proof that $\mathbf{S}_{n} \notin \mathcal{J}$. Indeed, let us say that there is an interpretation of variables of $\Phi_{n}$ in some $\mathbf{T} \in \mathcal{J}$ such that the hypothesis of $\Phi_{n}$ is true. Let $e_{j}$ denote the value of $x_{j} v_{j}$. Then any strong subset containing $e_{j}$ also contains $e_{j+1}$ (where addition in the subscript is modulo $n+1$ ). Since $\mathbf{T}$ is $T_{0}$ with respect to strong subsets, Lemma 2.1 implies that $e_{0}=e_{1}=\cdots=e_{n}$, as required. So $\Sigma$ satisfies property 1 of Lemma 5.7 .

To construct the semigroups $\mathbf{T}_{m}$ of Lemma 5.7 we use the methods of Section 5

For $n \in \mathbb{N}$, let $X_{n}:=\left\{a_{j}, b_{j}, c_{j}, d_{j}: 0 \leq j \leq n\right\}$. We now define a clean congruence on $\mathbf{F}_{3,1}\left(X_{n}\right)$ (which is simply the $X_{n}$-generated relatively free algebra in the variety generated by $\left.\mathbf{C}_{3,1}\right)$. Let $\theta$ be the equivalence relation generated by the following identifications (subtraction in the first subscript of an element is to be taken modulo $n+1)$ :

$$
a_{j} c_{j}=b_{j} c_{j}=b_{j} d_{j}=a_{j-1} d_{j-1}
$$

for each $0 \leq j \leq n$. Observe that these identifications correspond to the nonzero products in the series of semigroups used in Section 2, It is clear that $\theta$ is a stable congruence on $\mathbf{F}_{3,1}\left(X_{n}\right)$, and moreover property (函) (preceding Lemma [5.2) holds. We let $e_{j}$ denote the $\theta$-class of the element $a_{j} c_{j}$. The elements $e_{j}$ will be called special, while all other nonzero elements will be called ordinary elements. Ordinary elements correspond to the singleton congruence classes of $\theta$.

Lemma 6.1. $\mathbf{G}_{3,1}\left(X_{n}, \theta\right) \notin \mathcal{J}$.

Proof. $\mathbf{G}_{3,1}\left(X_{n}, \theta\right)$ clearly fails $\Phi_{n}$ under $x_{i} \mapsto a_{i}, v_{i} \mapsto c_{i}, u_{i} \mapsto b_{i}$ and $y_{i} \mapsto d_{i}$. 
There is an obvious automorphism of $\mathbf{G}_{3,1}\left(X_{n}, \theta\right)$ defined by incrementing the value of the subscript of the generators modulo $n+1$. We denote this automorphism by $\sigma$.

For each $m \in \mathbb{N}$ put $n:=2 m+1$ and choose $\mathbf{T}_{m}:=\mathbf{G}_{3,1}\left(X_{n}, \theta\right)$. Lemma 6.1 shows that property $2(\mathrm{a})$ of Lemma 5.7 holds. Now we verify property $2(\mathrm{~b})$.

Let $a$ be a nonzero element of $\mathbf{G}_{3,1}\left(X_{n}, \theta\right)$. Now recall that $D_{\mathbf{G}_{3,1}\left(X_{n}, \theta\right)}(a)$ is the subset of $X_{n}$ of generators dividing $a$ in $\mathbf{G}_{3,1}\left(X_{n}, \theta\right)$. Let $I$ be the set of all numbers $j$ such that $j$ is the subscript of an element of $D_{\mathbf{G}_{3,1}\left(X_{n}, \theta\right)}(a)$. If $a$ is a nontrivial $\theta$-class (equivalently, a special element), then $|I|=2$; otherwise we have $|I| \leq 2$. It follows that since $n=2 m+1$, any $m$-generated subsemigroup of $\mathbf{T}_{m}=\mathbf{G}_{3,1}\left(X_{n}, \theta\right)$ is a subsemigroup of $\mathbf{G}_{3,1}\left(Z_{n, j}, \theta\right)$, where $0 \leq j \leq n$ and $Z_{n, j}$ is the subset of $X_{n}$ consisting of all generators whose subscript is not $j$. We will show that $\mathbf{G}_{3,1}\left(Z_{n, j}, \theta\right)$ is contained in the quasivariety of $\mathbf{C}_{3,1}$. By applying $\sigma$ sufficiently many times to $\mathbf{G}_{3,1}\left(X_{n}, \theta\right)$, it suffices to restrict to $j=0$, and we will further abbreviate $Z_{n, 0}$ to $Z_{n}$.

Lemma 6.2. For all $n \in \mathbb{N}$, we have $\mathbf{G}_{3,1}\left(Z_{n}, \theta\right) \in \mathbb{Q}\left(\mathbf{C}_{3,1}\right)$.

Proof. First we fix some notation. Let $\mathbf{G}$ abbreviate $\mathbf{G}_{3,1}\left(Z_{n}, \theta\right)$ and let $\mathbf{F}$ abbreviate $\mathbf{F}_{3,1}\left(Z_{n}\right)$. We use the natural maps of Lemma 5.3 to witness the separation condition of Lemma 1.6 with respect to $\mathbf{C}_{3,1}$.

Let $x$ and $y$ be distinct elements of $B$. If one of $x$ and $y$ is 0 , then $\nu_{Z_{n}}$ separates $x$ and $y$. From now on we assume that neither $x$ nor $y$ equals 0 .

Case 1 (One of $x$ or $y$ is ordinary). Without loss of generality assume that $x$ is ordinary and that if $y$ is also ordinary, then either $|x| \neq|y|$ or both $|x|=|y|$ and $D_{\mathbf{G}}(y) \nsubseteq D_{\mathbf{G}}(x)$. Put $S:=D_{\mathbf{G}}(x)$, which is stable by Lemma 5.2 (using $w:=x$ ). The inequality $\nu_{S}(x) \neq \nu_{S}(y)$ is clear.

Case 2 (Both $x, y$ are special). Up to symmetry, we may assume that $x=e_{k}$ for some $k>0$ and that

$$
y \notin\left\{e_{k}, e_{k+1}, \ldots, e_{n}\right\} .
$$

Let $T \subseteq Z_{n}$ consist of all elements whose subscript $\ell$ has $\ell \geq k$, and let $S:=$ $T \cup\left\{a_{k-1}, d_{k-1}\right\}$. It is easy to see that $S=D_{\mathbf{G}}\left(\left\{e_{k}, e_{k+1}, \ldots, e_{n-1}\right\}\right)$. We claim that $S$ is stable. Let $u, v$ be two distinct $\theta$-related elements of $\mathbf{F}$ with $u / \theta \in \operatorname{Sg}^{\mathbf{G}}(S)$. The definition of $\theta$ and the choice of $S$ show that $u / \theta$ (which equals $v / \theta$ ) is of the form $e_{\ell}$ for some $\ell \geq k$. But then $D_{\mathbf{F}}(v) \subseteq S$, showing that $S$ is stable by Lemma 5.1. We now have $\nu_{Z}(x) \neq \nu_{Z}(y)$, as required.

This exhausts the possibilities for $x \neq y$ and so completes the proof.

This also verifies the property $2(\mathrm{~b})$ of Lemma 5.7 Theorem $1.11_{\text {i) }}$ now follows.

\section{Proof of Theorem 1.1 II $)(\mathrm{III})$}

In this section we prove Theorem $1.1_{\text {ii } \vee(\text { iii })}$. We fix the number $k \in \mathbb{N}$ and use Lemma 5.7, where $Q=\mathcal{O} \vee \mathcal{F}_{k}$. The proof is very similar to that of the previous section and we redefine much of the notation used there. 
7.1. The semigroups $\mathbf{T}_{m}$. For each number $n \in \mathbb{N}$, we let $s=s(n, k):=(2 n+1) k$. Next we define a set $X_{n}=\left\{a_{j} \mid 0 \leq j \leq s\right\}$. Now let $\operatorname{Seq}_{n}$ denote the set of all nonrepeating sequences $\sigma:\{0,1, \ldots, 2 n+1\} \rightarrow\{0,1,2, \ldots, s\}$ (so $\sigma(i)=\sigma(j) \Rightarrow$ $i=j)$.

Now we define a set of generators:

$$
Y_{n}:=X_{n} \cup\left\{b_{i}^{\sigma} \mid \sigma \in \operatorname{Seq}_{n}, 0 \leq i \leq n\right\} .
$$

For notational convenience, let id denote the sequence $0,1,2, \ldots, 2 n+1$ and define $e:=a_{0} b_{0}^{\text {id }}$ and $f:=a_{2 n+1} b_{n}^{\text {id }}$.

We use the construction $\mathbf{F}_{3,1}\left(Y_{n}\right)$ of Section 5 , For each sequence $\sigma \in \operatorname{Seq}_{n}$ we make the following identifications between length 2 words:

$$
\begin{aligned}
e & \equiv a_{\sigma(0)} b_{0}^{\sigma}, \\
a_{\sigma(1)} b_{0}^{\sigma} & \equiv a_{\sigma(2)} b_{1}^{\sigma}, \\
& \vdots \\
a_{\sigma(2 j+1)} b_{j}^{\sigma} & \equiv a_{\sigma(2 j+2)} b_{j+1}^{\sigma}, \\
& \vdots \\
a_{\sigma(2 n-1)} b_{n-1}^{\sigma} & \equiv a_{\sigma(2 n)} b_{n}^{\sigma}, \\
a_{\sigma(2 n+1)} b_{n}^{\sigma} & \equiv f .
\end{aligned}
$$

Let $\theta$ be the equivalence relation generated by the relation $\equiv$ on $\mathbf{F}_{3,1}\left(Y_{n}\right)$. Since the sequences in Seq are nonrepeating, it is easy to verify that the only extra identifications beyond those described above are $a_{\sigma(i)} b_{i}^{\sigma} \equiv a_{\gamma(i)} b_{i}^{\gamma}$ for $i=0,2 n$ and $\sigma, \gamma \in$ Seq. Also, the only nontrivial blocks are sets of length 2 words, each involving two distinct letters, so $\theta$ is a clean congruence on $\mathbf{F}_{3,1}\left(Y_{n}\right)$ satisfying property (因). For $u \in \mathbf{G}_{3,1}\left(Y_{n}, \theta\right)=\mathbf{F}_{3,1}\left(Y_{n}\right) / \theta$, we use $[u]$ to abbreviate $u / \theta$. Note that $[e]$ and $[f]$ are distinct elements of $\mathbf{G}_{3,1}\left(Y_{n}, \theta\right)$.

Now $\theta$ has precisely two congruence classes of size more than 2: namely the elements $[e]$ and $[f]$. There are also a number of congruence classes of size 2, corresponding to each of the other identifications made: these are of the form

$$
\left[a_{\sigma(2 j+1)} b_{j}^{\sigma}\right]=\left\{a_{\sigma(2 j+1)} b_{j}^{\sigma}, a_{\sigma(2 j+2)} b_{j+1}^{\sigma}\right\},
$$

for $j=0,1, \ldots, n-1$ and $\sigma \in$ Seq. Such elements will be called type $j$ elements. The elements $[e],[f]$ and the type $j$ elements for $j=0,1, \ldots, n-1$ will be called the special elements. Nonzero elements that are not special will be called ordinary elements. The following lemma is now obvious.

Lemma 7.1. Let $w$ be a nongroup element of $\mathbf{G}_{3,1}\left(Y_{n}\right)$.

- If $w=[e]$, then $D(w) \cap\left\{b_{j}^{\sigma} \mid 0 \leq j \leq n, \sigma \in \operatorname{Seq}_{n}\right\}=\left\{b_{0}^{\sigma} \mid \sigma \in \operatorname{Seq}_{n}\right\}$.

- If $w=[f]$, then $D(w) \cap\left\{b_{j}^{\sigma} \mid 0 \leq j \leq n, \sigma \in \operatorname{Seq}_{n}\right\}=\left\{b_{n}^{\sigma} \mid \sigma \in \mathrm{Seq}_{n}\right\}$.

- If $w$ is of type $j$, then $D(w) \cap\left\{b_{j}^{\sigma} \mid 0 \leq j \leq n, \sigma \in \mathrm{Seq}_{n}\right\}=\left\{b_{j}^{\sigma}, b_{j+1}^{\sigma}\right\}$ for some $\sigma \in \mathrm{Seq}_{n}$.

- If $w$ is ordinary, then $\left|D(w) \cap\left\{b_{j}^{\sigma} \mid 0 \leq j \leq n, \sigma \in \operatorname{Seq}_{n}\right\}\right| \leq 2$.

For any $m \in \mathbb{N}$, put $n=2 m+1$ (so that $s=(4 m+3) k$ ). We define the semigroup $\mathbf{T}_{m}:=\mathbf{G}_{i, p}\left(Y_{n}, \theta\right)$. 
7.2. The split quasi-identities $\Sigma_{k}$. For any $n \in \mathbb{N}$, let $F_{n}$ denote the following formulæ, where the $x_{j}$ and $y_{j}^{\sigma}$ are all distinct variables:

$$
\begin{aligned}
\underset{\substack{\sigma \in \operatorname{seq}_{n}, 0 \leq j \leq n-1}}{\&} x_{\sigma(2 j+1)} y_{j}^{\sigma} \approx x_{\sigma(2 j+2)} y_{j+1}^{\sigma} & \& \quad \underset{\sigma, \tau \in \operatorname{Seq}_{n}}{\&} x_{\sigma(0)} y_{0}^{\sigma} \approx x_{\tau(0)} y_{0}^{\tau} \\
& \& \underset{\sigma, \tau \in \operatorname{Seq}_{n}}{\&} x_{\sigma(2 n+1)} y_{n}^{\sigma} \approx x_{\tau(2 n+1)} y_{n}^{\tau}
\end{aligned}
$$

and abbreviate the (split) quasi-identity $F_{n} \rightarrow x_{0} y_{0}^{\text {id }} \approx x_{2 n+1} y_{n}^{\text {id }}$ by $\Phi_{n}$. (It is clear from the third part of the definition of $F_{n}$ that any element of $\mathrm{Seq}_{n}$ would do in place of id.)

We let $\Sigma_{k}:=\left\{\Phi_{n} \mid n \in \mathbb{N}\right\}$. The following lemma establishes hypotheses 1 and 2(a) of Lemma 5.7 .

Lemma 7.2. For each $n \in \mathbb{N}$, O $\vee \mathcal{F}_{k} \models \Phi_{n}$ but $\mathbf{G}_{i, p}\left(Y_{n}, \theta\right) \not \models \Phi_{n}$.

Proof. For the first statement, it suffices to prove that $\mathcal{O} \models \Phi_{n}$ and $\mathcal{F}_{k} \models \Phi_{n}$. For $\mathcal{O}$, let us assume that we have an interpretation $\theta$ of the variables in some semigroup $\mathbf{S} \in \mathcal{O}$. Let $\epsilon$ and $\phi$ denote the values of $\theta\left(a_{0}\right) \theta\left(b_{0}^{\text {id }}\right)$ and $\theta\left(a_{2 n+1}\right) \theta\left(b_{n}^{\text {id }}\right)$, respectively. By Lemma 3.1 we have, up to symmetry, a total right-compatible preorder $\leq$ (with $\geq$ and $\equiv$ defined in the obvious way) on $\mathbf{S}$ with $\epsilon \geq \phi$ (and our goal is to show that we have equality). Let us denote $\alpha_{j}:=\theta\left(a_{j}\right)$ and $\beta_{j}^{\sigma}:=\theta\left(b_{j}^{\sigma}\right)$. Evidently, there is a permutation $\sigma$ of $\{0,1, \ldots, 2 n+1\}$ such that $\alpha_{\sigma(0)} \leq \alpha_{\sigma(1)} \leq \cdots \leq \alpha_{\sigma(2 n+1)}$. Now $\sigma$ is an element of $\mathrm{Seq}_{n}$, so we have

$$
\begin{aligned}
\epsilon & =\alpha_{\sigma(0)} \beta_{0}^{\sigma} \\
\leq \alpha_{\sigma(1)} \beta_{0}^{\sigma} & =\alpha_{\sigma(2)} \beta_{1}^{\sigma} \\
\vdots & \\
\leq \alpha_{\sigma(2 j+1)} \beta_{j}^{\sigma} & =\alpha_{\sigma(2 j+2)} \beta_{j+1}^{\sigma} \\
\vdots & \\
\leq \alpha_{\sigma(2 n-1)} \beta_{n-1}^{\sigma} & =\alpha_{\sigma(2 n)} \beta_{n}^{\sigma} \\
\leq \alpha_{\sigma(2 n+1)} \beta_{n}^{\sigma} & =\phi
\end{aligned}
$$

showing that $\epsilon \leq \phi$, and hence $\epsilon \equiv \phi$. Now as $\leq$ was an arbitrary total rightcompatible preorder and $\mathbf{S} \in \mathcal{O}$, Lemma 3.1 shows that $\epsilon=\phi$, as required.

Now we must show that $\mathcal{F}_{k}=\Phi_{n}$. Let $\mathbf{S}$ be any $k$-element groupoid and let $\theta$ be a variable assignment into $\mathbf{S}$. We keep the same notation as in the previous case. Now the set $\left\{a_{j} \mid 0 \leq j \leq s\right\}$ has size $(2 n+1) k+1$, so it must have some $(2 n+2)$-element subset $X$ with the property that $|\theta(X)|=1$. Let $\sigma \in \operatorname{Seq}_{n}$ be any sequence with range $X$. So for every $i, j$ we have $\alpha_{\sigma(i)}=\alpha_{\sigma(j)}$ from which we easily obtain $\epsilon=\phi$.

For the second statement of the lemma, consider the variable assignment into $\mathbf{G}_{i, p}\left(Y_{n}, \theta\right)$ given by $x_{j} \mapsto a_{j}$ and $y_{j}^{\sigma} \mapsto b_{j}^{\sigma}$. This assignment gives the premise $F_{n}$ of $\Phi_{n}$ a positive truth value. However the expressions $x_{0} y_{0}^{\text {id }}$ and $x_{2 n+1} y_{n}^{\text {id }}$ take the values $[e]$ and $[f]$ respectively, contradicting the conclusion of $\Phi_{n}$. So $\phi$ causes $\Phi_{n}$ to fail on $\mathbf{G}_{i, p}\left(Y_{n}, \theta\right) \backslash\{0\}$. 
7.3. Property 2(b). Recall that $n=2 m+1$. To satisfy hypothesis 2(b) of Lemma 5.7 we need to show that for each $m \in \mathbb{N}$, the $m$-generated subalgebras of $\mathbf{T}_{m}=$ $\mathbf{G}_{3,1}\left(Y_{n}, \theta\right)$ are contained in $\operatorname{ISP}\left(\mathbf{C}_{3,1}\right)$.

For each $\ell=0,1, \ldots, n$, let $\mathbf{B}_{\ell}$ denote the subsemigroup of $\mathbf{G}_{3,1}\left(Y_{n}, \theta\right)$ generated by the set $Z_{n, \ell}:=Y_{n} \backslash\left\{b_{\ell}^{\sigma} \mid \sigma \in\right.$ Seq $\}$. In other words, $\mathbf{B}_{\ell}=\mathbf{G}_{3,1}\left(Z_{n, \ell}, \theta\right)$. We also let $\mathbf{F}$ abbreviate $\mathbf{F}_{3,1}\left(Z_{n, \ell}\right)$. By Lemma 7.1, an $m$-generated subalgebra of $\mathbf{G}_{3,1}\left(Y_{n}, \theta\right)$ lies inside $\mathbf{B}_{\ell}$ for some $\ell$.

For the remainder of this proof we use $[u]$ to denote the equivalence class of $u$ in $F$ modulo the restriction of $\theta$ to $\mathbf{F}=\mathbf{F}_{3,1}\left(Z_{n, \ell}\right)$ (instead of $u$ in $F_{3,1}\left(Y_{n}\right)$ modulo $\theta$ ). Under this notation, special elements of type $\ell-1$ and type $\ell+1$ are singleton congruence classes (with only one of these making sense if $\ell=0$ or $n$ ). For the remainder of the proof it is convenient to consider these elements as ordinary elements (so there will be no special elements of any of the types $\ell-1, \ell, \ell+1$, whenever these make sense).

We now verify the separation condition of Lemma 1.6 for $\mathbf{B}_{\ell}$ with respect to $\mathbf{C}_{3,1}$. Since the number $\ell$ is fixed in the remaining arguments, we write $\mathbf{B}$ in place of $\mathbf{B}_{\ell}$.

Case $0.0 \in\{x, y\}$.

The set $Z_{n, \ell}$ of generators is stable and its natural map separates $x$ and $y$.

Throughout the remainder of the proof we assume that neither $x$ nor $y$ is equal to 0 .

Case 1. One of $x, y$ is ordinary.

Without loss of generality we may assume that $x$ is ordinary, and that if $y$ is ordinary, then $|x| \neq|y|$ or both $|x|=|y|$ and $D_{\mathbf{B}}(y) \nsubseteq D_{\mathbf{B}}(x)$. Put $S:=D_{\mathbf{B}}(x)$, which is stable by Lemma 5.2. The inequality $\nu_{S}(x) \neq \nu_{S}(y)$ is now clear.

Case 2. $x$ and $y$ are special, and one of $x$ and $y$ is of type $j$ (some $j \in\{0, \ldots$, $\ell-2, \ell+2, \ldots, n-1\})$.

Without loss of generality, say that $x$ is of type $j$, and let $S:=D_{\mathbf{B}}(x)$. We prove that $S$ is stable. Say that $u$ and $v$ are distinct elements of $\mathbf{F}$ with the property that $u \theta v$ and $[u] \in \operatorname{Sg}^{\mathbf{B}}(S)$. Now it is easy to see that there is only one special element in $\operatorname{Sg}^{\mathbf{B}}(S)$ and that is $x$. As $x$ is type $j$ it is a two-element congruence class and so we have $x=\{u, v\}$. As $S=D_{\mathbf{B}}(x) \supseteq D_{\mathbf{F}}(v)$, Lemma 5.1 shows that $S$ is stable. Now we have $\nu_{S}(x)=a^{i-1} \neq a^{i p+|y|}=\nu_{S}(y)$.

Case 3. $\{x, y\}=\{[e],[f]\}$. (This case is only possible if $\ell \notin\{0, n\}$.) Up to symmetry we may assume that $x=[e]$. Define $S:=Z_{n, \ell} \backslash\left\{b_{\ell}^{\sigma} \mid j>\ell, \sigma \in\right.$ Seq $\}$. Now the only special elements in $\langle S\rangle$ are $[e]$ and the elements of type $j$ for $j<\ell$. Let $u, v$ be two distinct $\theta$-related elements of $\mathbf{F}$ with $u \in \operatorname{Sg}^{\mathbf{F}}(S)$. So $[u]$ is either $[e]$ or a type $j$ element with $j<\ell$. Hence $D_{\mathbf{F}}(v)$ does not contain $b_{\ell}^{\sigma}$ for any $j>\ell$. So $D_{\mathbf{F}}(v) \subseteq S$, showing that $S$ is stable by Lemma 5.1. We have $\nu_{A}(x)=a^{i-1} \neq$ $a^{i p+i-1}=\nu_{A}(y)$.

Since this exhausts the possible choices of $x$ and $y$, we conclude by Lemma 1.6 that $\mathbf{B}_{\ell} \in \mathbb{Q}\left(\mathbf{C}_{3,1}\right)$.

Theorem 1.1 (ii) $\vee($ iii) now follows from Lemma 5.7 ,

The proofs in Section 6 and the present Section 7 may give the impression that $\mathbf{C}_{3,1}$ might be proved not finitely q-based using any nonfinitely based system of 
split quasi-identities. However this is not the case. The quasi-identity

$$
\Psi_{n}:=\underset{0 \leq j \leq 2 n+2}{\&} x z_{j} \approx y z_{j-1} \rightarrow x z_{0} \approx x z_{1} \approx \cdots \approx x z_{2 n+2}
$$

(where subtraction is taken modulo $2 n+3$ ) underlies the construction $\mathbf{S}_{n}$ of Section 3 and is obviously split. However $\Psi_{n}$ follows - for any $n$-from the single quasiidentity $x z_{0} \approx y z_{1} \quad \& \quad x z_{1} \approx y z_{2} \rightarrow x z_{1} \approx y z_{1}$, which is routinely seen to hold on the 3-nilpotent semigroup $\mathbf{C}_{3,1}$.

\section{Monoids AND LOCAL PROperties}

In this short section we observe some elementary facts showing how to extend our main result to other classes.

In this section, $\mathbf{S}^{(1)}$ will stand for the semigroup resulting by adjoining an identity element to $\mathbf{S}$, even if the semigroup $\mathbf{S}$ already has an identity element. If $\mathcal{Q}$ is a quasivariety of semigroups, then we let $Q_{\text {mon }}$ denote the class of all monoids (in the type $\langle 2,0\rangle$ ) whose semigroup reducts are in $\mathcal{Q}$. It is not hard to verify that $\mathcal{Q}_{\text {mon }}$ is a quasivariety of monoids.

Let $Q$ be a semigroup quasivariety. We say that a semigroup $\mathbf{S}$ is locally- $Q$ if for every idempotent $e \in S$ we have $e \mathbf{S} e \in \mathcal{Q}$.

Lemma 8.1. Let $\mathbf{S}$ be a semigroup and $\mathcal{Q}$ a quasivariety. Suppose that for every $n \in \mathbb{N}$ there is a semigroup $\mathbf{T}_{n}$ with $\mathbf{T}_{n} \notin Q$ but whose $n$-generated subsemigroups are contained in $\mathbb{I S P}(\mathbf{S})$. Further assume that $\mathbf{S}^{(1)} \in \mathcal{Q}$. Let $\mathbf{M}$ be $\mathbf{S}^{(1)}$ considered as a monoid of type $\langle 2,0\rangle$. The following hold:

(1) $\mathbf{S}^{(1)}$ is INFQB relative to being locally-Q;

(2) $\mathbf{M}$ is INFQB relative to membership in $Q_{\text {mon }}$.

Proof. For (1), say that $\mathbf{A}$ is locally- $Q$ and generates a quasivariety containing $\mathbf{S}^{(1)}$. Now an $n$-generated subsemigroup (or submonoid) of $\mathbf{T}_{n}^{(1)}$ is a submonoid of $\mathbf{U}_{n}^{(1)}$ for some $n$-generated subsemigroup $\mathbf{U}_{n}$ of $\mathbf{T}_{n}$. As $\mathbf{U}_{n} \in \mathbb{Q}(\mathbf{S})$ we also have $\mathbf{U}_{n}^{(1)} \in \mathbb{Q}\left(\mathbf{S}^{1}\right)$. However $\mathbf{T}_{n}^{(1)}$ is not locally in $\mathcal{Q}$ since using $e:=1$ we have $\mathbf{T}_{n}^{(1)}=e \mathbf{T}_{n}^{(1)} e$ and $\mathbf{T}_{n}$, whence $\mathbf{T}_{n}^{(1)}$, fails some quasi-identity of $\mathcal{Q}$. Hence $\mathbf{A}$ is nonfinitely q-based by Lemma 1.7 .

The proof of (2) is very similar.

The monoid version of Theorem 1.1 now follows from the semigroup version.

\section{REFERENCES}

1. S. Burris and H.P. Sankappanavar, A Course in Universal Algebra, Graduate Texts in Mathematics 78, Springer-Verlag, Berlin-Heidelberg-New York, 1980. MR648287 (83k:08001)

2. D.M. Clark, B.A. Davey, M.G. Jackson and J.G. Pitkethly, The axiomatisability of topological prevarieties, Adv. Math. 218 (2008), 1604-1653. MR2419934

3. A.H. Clifford and G.B. Preston, The Algebraic Theory of Semigroups. Vols. I, II. Mathematical Surveys, No. 7, American Mathematical Society, Providence, R.I. 1961, 1967. MR0132791 (24:A2627) MR0218472 (36:1558)

4. D.F. Cowan, N.R. Reilly, P.G. Trotter and M.V. Volkov, The finite basis problem for quasivarieties and pseudovarieties generated by regular semigroups I. Quasivarieties generated by regular semigroups, J. Algebra 267 (2003), 635-653. MR2003345 (2004m:20108)

5. J. Dénes and A.D. Keedwell, Latin Squares and Their Applications, Academic Press, New York-London, 1974. MR 0351850 (50:4338)

6. V.A. Gorbunov, Algebraic Theory of Quasivarieties, Consultants Bureau, New York, 1998. MR.1654844 (2001a:08004) 
7. M. Jackson, Small Semigroup Related Structures with Infinite Properties, Ph.D. thesis, University of Tasmania, 1999.

8. M. Jackson, Flat algebras and the translation of universal Horn logic to equational logic, J. Symbolic Logic. 73 (2008), 90-128. MR2387934

9. M. Jackson, Residual bounds for compact totally disconnected algebras, Houston J. Math. 34 (2008), 33-67. MR2383696

10. M. Jackson and T. Stokes, Algebras of partial maps, to appear in the Proceedings of the Sydney Conference on Semigroup Theory and Related Mathematics.

11. D.J. Kleitman, B.R. Rothschild and J.H. Spencer, The number of semigroups of order n, Proc. Amer. Math. Soc. 55 (1976), 227-232. MR 0414380 (54:2483)

12. V. Koubek and V. Rödl, Note on the number of monoids of order n, Comment. Math. Univ. Carolin. 26 (1985), 309-314. MR803927 (87a:05016)

13. J. Lawrence and R. Willard, On finitely based groups and nonfinitely based quasivarieties, J. Algebra 203 (1998), 1-11. MR1620693 (99g:20047)

14. A.I. Mal'cev, On the immersion of the associative systems in groups. I, Mat. Sb. 6 (1939), 331-336 [Russian].

15. A.I. Mal'cev, On the immersion of the associative systems in groups. II, Mat. Sb. 8 (1940), 251-264 [Russian].

16. A.I. Mal'cev, On the general theory of algebraic systems, Mat. Sb. 35 (1954), 3-20 [Russian]. MR.0065533(16:440e)

17. S.W. Margolis and M.V. Sapir, Quasi-identities of finite semigroups and symbolic dynamics, Israel J. Math. 92 (1995), 317-331. MR.1357761 (96i:20075)

18. V.L. Murskiř, The existence of a finite basis of identities, and other properties of "almost all" finite algebras, Problemy Kibernet. 30 (1975), 43-56 [Russian]. MR0401606 (53:5433)

19. S. Oates and M. B. Powell, Identical relations in finite groups, J. Algebra 1 (1964), 11-39. MR0161904 (28:5108)

20. A.Yu. Ol'shanskiǐ, Conditional identities in finite groups, Sibirsk. Mat. Zh. 15 (1974), 14091413 [Russian; English translation in Siberian Math. J. 15 (1975), 1000-1003]. MR0367068 (51:3310)

21. M. Petrich, Inverse Semigroups, Wiley Interscience, New York, 1984. MR 752899 (85k:20001)

22. V.B. Repnitskiǐ and A.S. Vernitskiǐ, Semigroups of order-preserving mappings, Comm. Algebra 28 (2000), 3635-3641. MR1767577 (2001b:20109)

23. M.V. Sapir, On the quasivarieties generated by finite semigroups, Semigroup Forum 20 (1980), 73-88. MR.572536 (81g:08016)

24. M.V. Sapir, An implication characterisation of prevarieties of semigroups and rings, Ural. Gos. Univ. Mat. Zap. 13, no. 1 (1982), 121-132 [Russian]. MR694237 (84i:20063)

25. M.V. Sapir, Semigroup Quasivarieties, Ph.D. thesis, Moscow State Pedagogical Institute, 1983 [Russian].

26. B.M. Schein, A system of axioms for semigroups embeddable in generalized groups, Doklady Akad. Nauk SSSR 134 (1960), 1030-1034 [Russian; English translation in Soviet Math. Doklady 1 (1960), 1180-1183]. MR0141719 (25:5116)

27. B.M. Schein, Embedding semigroups in generalized groups, Mat. Sb. 55 (1961), 379-400 [Russian; English translation in Translations of the Amer. Math. Soc. (2) 139 (1962), 164-176]. MR 0139673 (25:3104)

28. B.M. Schein, An Abstract Theory of Semigroups of One-to-One Transformations, Ph.D. thesis, Saratov State University, 1962 [Russian].

29. B.M. Schein, Relation algebras and function semigroups, Semigroup Forum 1 (1970), 1-62. MR0285638 (44:2856)

30. B.M. Schein, Subsemigroups of inverse semigroups, Matematiche (Catania) 51 (1996), 205227. MR:1485712 (98m:20078)

31. B.M. Schein, On finite semigroups embeddable in inverse semigroups, Semigroup Forum 62 (2001), 329-330. MR 1831515

32. L.N. Shevrin and M.V. Volkov, Identities of semigroups, Izv. Vyssh. Uchebn. Zaved. Mat. (1985), no. 11, 3-47 [Russian; English translation in Soviet Math. (Iz. VUZ) 29 (1985), no. 11, 1-64]. MR829099 (87f:20094)

33. J.D.H. Smith, Mal'cev Varieties, Lecture Notes in Mathematics 554, Springer-Verlag, BerlinHeidelberg-New York, 1976. MR0432511 (55:5499) 
34. A.S. Vernitskiı̌, The finite basis problem for the semigroups of order-preserving mappings, Proc. Roy. Soc. Edinburgh Sect. A 129 (1999), 641-647. MR1693605 (2000e:20092)

35. M.V. Volkov, The finite basis problem for finite semigroups, Sci. Math. Jpn. 53 (2001), 171199. MR1821612 (2002a:20069)

Department of Mathematics and Statistics, La Trobe University, Victoria 3086, AusTRALIA

E-mail address: M.G.Jackson@latrobe.edu.au

Department of Mathematics, Ural State University, Ekaterinburg 620083, Russia

E-mail address: Mikhail.Volkov@usu.ru 